[45] H. Bénard, Ann. Chim. Phys. 23, 62 (1901).

[46] H. L. Koschmieder in R. J. Donnelly, R. Herman u. I. Prigogine Non-Equilibrium Thermodynamics, Variational Techniques and Stability. University of Chicago Press, Chicago 1966, S. 172.
[47] M. Turing, Trans. Roy. Soc. (London) B 237, 37 (1952).

[48] A. M. Zhabotinskii, Biofizika 9, 306 (1964).

[49] A, M. Zhahotinskii, Dokl. Akad. Nauk SSSR 157, 392 (1964).

\title{
Molekulare Hysterese und ihre kybernetische Bedeutung
}

\author{
Von Eberhard Neumann ${ }^{[*]}$
}

Die allgemeinen Grundlagen für die thermodynamische Analyse von Hystereseerscheinungen in Lösungen und Suspensionen polyelektrolytischer Systeme werden anhand von Beispielen molekularer Hysterese in Biopolymeren und Membranen dargelegt. Die fundamentale kybernetische Bedeutung metastabiler Zustände und molekularer Hysterese für die physikalische Interpretation von Lebenserscheinungen wie Gedächtnisaufzeichnung und biologische Rhythmen wird diskutiert.

\section{Einleitung}

„Das Ziel biologischer Forschung - so glaube ich - ist letztlich die, Übersetzung der Lebenserscheinungen in sinnvolle physikalische Grundkonzepte." - Aharon Katchalsky ${ }^{[* *]}$

Die moderne physikalische Chemie biologischer Makromoleküle und Membranen hat ihre Wurzeln in der klassischen Kolloidchemie. Besonders wegen ihrer kolloidähnlichen Eigenschaften wurden hochmolekulare Bestandteile biologischer Zellen und Gewebe als Biokolloide bezeichnet. Man hat jedoch bald erkannt, daß viele der so klassifizierten Zellkomponenten nicht kolloidale Aggregate kleiner Moleküle, sondern große Moleküle kolloidaler Dimensionen sind ${ }^{[1]}$.

In der Mehrzahl sind biologische Makromoleküle lineare, langkettige Polymere, deren Monomer-Einheiten unter physiologischen Bedingungen des $\mathrm{pH}-$ Werts (etwa $\mathrm{pH} 7$ bis 8 ) und der Ionenstärke (etwa $0.15 \mathrm{~mol} / \mathrm{l}$ ) elektrisch geladen sind. So zählen zum Beispiel alle Nucleinsäuren und sauren Mucopolysaccharide elektrochemisch zu den Polyelektrolyten. Auch die „Oberflächen“ vieler Strukturproteine, Enzyme und Biomembranen sind dicht mit elektrisch geladenen Gruppen bedeckt und können deshalb als polyelektrolytische Systeme betrachtet werden.

Den elektrochemischen Eigenschaften von Biopolymeren und biopolyelektrolytischen Molekülorganisationen wie Membranen wird neuerdings wachsende Aufmerksamkeit gewidmet. Denn eine Reihe der die spezifische Organisation und Funktion dieser Zellkomponenten betreffenden Merkmale beruht auf den elektrischen Wechselwirkun-

[*] Priv.-Doz. Dr. E. Neumann

Max-Planck-Institut für Biophysikalische Chemie

34 Göttingen-Nikolausberg. Postfach 968

[**] „Wer ist's?“", Nachr. Chem. Tech. 20 (13), 247 (1972). gen, die sie untereinander und mit ihrer Umgebung ausüben. So können Veränderungen in Struktur und Funktion solcher Systeme durch elektrische und elektrochemische Einflïsse der Umgebung gesteuert und geregelt werden ${ }^{[2]}$. Dieser kybernetische Gesichtspunkt gewinnt besonderes Interesse im Zusammenhang mit einer weiteren bemerkenswerten Eigenschaft vieler „Biokolloide“: der Fähigkeit zu langlebigen metastabilen Zuständen.

Es ist eine bekannte Erfahrung der Kolloidchemie, daß in Kolloiden hoher elektrischer Aufladung häufig thermodynamisch nicht stabile Zustände und irreversible Zustandsänderungen auftreten können ${ }^{[3]}$. Es sollte daher nicht überraschen, daß auch polyelektrolytische Organisationen von Biopolymeren und Biomembranen langlebige metastabile Zustände auszubilden vermögen. In einer Reihe von Fällen spiegeln sich nun solche Metastabilitäten in ausgeprägten Hystereseerscheinungen wider.

Das wohl vertrauteste Beispiel langlebiger Metastabilität begegnet uns in den Hystereseschleifen ferromagnetischer Stoffe. Aber auch in anderen Bereichen der Physik und Chemie sind Hysteresen weit verbreitet, so z. B. bei Schmelz-Kristallisations-Zyklen von Ammoniumhalogeniden oder bei Adsorptions-Desorptions-Prozessen an porösen Stoffen. Das hysteretische Verhalten wird in diesen letztgenannten Fällen makroskopisch mit Poren- und Domänstrukturen gedeutet ${ }^{[4]}$.

Das Wort „Hysterese“ ist von Ewing geprägt worden, um die mehrdeutige, von der Magnetisierungsvorgeschichte abhängige Reaktion von Magneten auf Änderungen des äußeren elektrischen Feldes zu beschreiben ${ }^{5]}$. Der Termi-

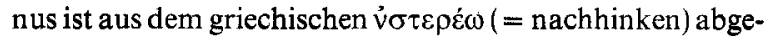
leitet; beispielsweise hinkt die magnetische Polarisation bei einem Magnetisierungs-Demagnetisierungs-Zyklus von Weicheisen der magnetischen Feldstärke nach.

Im Gegensatz zu den lange bekannten makroskopischen Hysteresen in und an kondensierten Phasen wurde mikro- 
skopische Hysterese einzelner Moleküle in homogener Lösung erst vergleichsweise spät entdeckt. Im Jahre 1956 fanden Cox, Jones, Marsh und Peacocke, daß die potentiometrische Säure-Base-Titration von ribosomaler Ribonucleinsäure (rRNA) keine Gleichgewichtskurve, sondern eine Hystereseschleife für den Protonenbindungsgrad als Funktion des $\mathrm{pH}$-Wertes ergibt ${ }^{[6]}$. Die intramolekulare Natur der rRNA-Hysterese konnte kürzlich nachgewiesen werden $^{[7]}$.

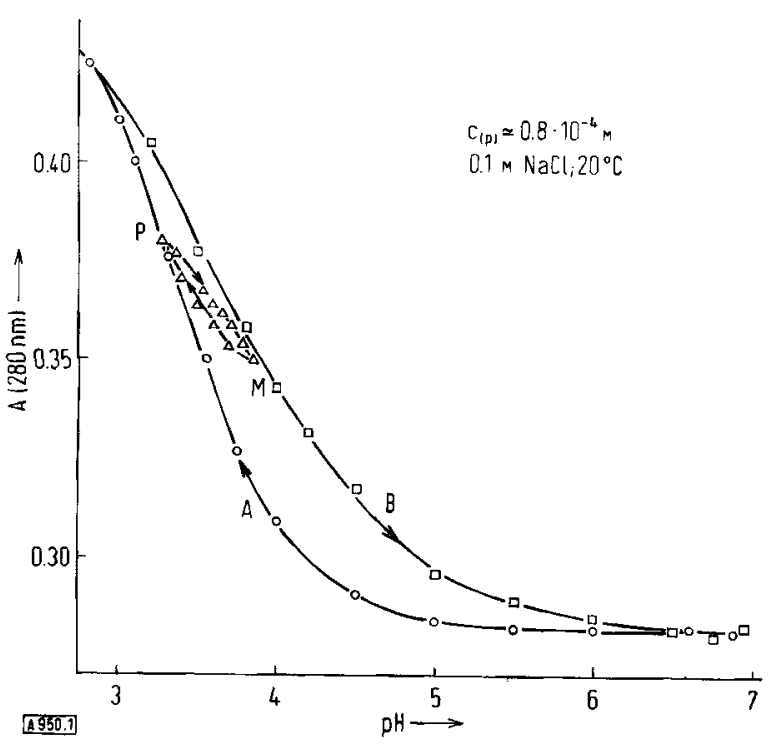

Abb. 1. Spektrophotometrische Titration ribosomaler RNA, Absorption (A) bei $280 \mathrm{~nm}$ als Funktion des pH-Werts. O: Säuretitration (Kurve A); D: anschließend Basetitration (Kurve B); $\Delta$ : Scanningschleife. Nach [7].

In Abbildung 1 ist das Ergebnis einer spektrophotometrischen Säure-Base-Titration von rRNA dargestellt. Die Hystereseschleife ist praktisch zeitunabhängig (siehe Abschnitt 2) und kann mehrfach ,umlaufen“ werden. Die Umlaufrichtung ist jedoch determiniert: die Titration einer neutralen Lösung mit Säure ergibt stets den unteren Kurvenast, die anschließende Titration der sauren Lösung mit Base führt immer entlang des oberen Asts der Grenzkurve. Innerhalb der Grenzschleife können Zwischenkurven erhalten werden, die den bekannten Scanning- oder Abtastkurven der magnetischen Hysterese ähnlich sind. Da diese Zwischenkurven intermediäre Systemzustände widerspiegeln, kann „Scanning“ im Prinzip zum Abtasten dieser Zwischenzustände benutzt werden. Die Analyse von Scanning-Kurven ist daher eine (neuartige) Methode, Strukturuntereinheiten in Biopolymeren wie rRNA in Lösung zu untersuchen ${ }^{[8]}$.

Zahlreiche Beispiele für Hysterese in Lösung oder Suspension sind inzwischen bekannt; weniger zahlreich sind hingegen Versuche, diese Nichtgleichgewichtserscheinungen $\mathrm{zu}$ analysieren und die physikalische Ursache der zugrundeliegenden Metastabilitäten $\mathrm{zu}$ ergründen. Tatsächlich sind Hysteresen von Physikern und Chemikern für lange Zeit als suspekte Kuriosität angesehen und offenbar gemieden worden. Die praktische Zeitunabhängigkeit stabiler Hysterese erschien als Widerspruch zur Richtungsabhängigkeit des Umlaufs. Gerichtetheit und Änderung mit der Zeit sind Kennzeichen irreversibler (natürlicher) Prozesse, wäh- rend Zeitunabhängigkeit ein Merkmal für den Grenzfall des Gleichgewichts (und des stationären Zustands) ist. Zeitunabhängige Irreversibilität, wie sie in langlebiger $\mathrm{Hy}$ sterese zum Ausdruck kommt, schien weder mit den Methoden der klassischen Thermostatik noch mit denen der Nichtgleichgewichtsthermodynamik zugänglich zu sein.

Es ist das Verdienst Aharon Katchalskys, nicht nur zur begrifflichen Klärung des scheinbaren Widerspruchs beigetragen, sondern auch die fundamentale Bedeutung von Metastabilität und Hysterese für zentrale Lebenserscheinungen wie zum Beispiel Erfahrungsgedächtnis oder biologische Rhythmen erkannt zu haben ${ }^{[9,10]}$.

Hysterese ist Ausdruck physikalischen Gedächtnisses, und bekanntlich werden die Hysteresezyklen ferromagnetischer und ferritischer Bauelemente in der Technologie der Rechenmaschinen zur Speicherung von Information benutzt.

In der Biologie ist der Mechanismus der Gedächtnisaufzeichnung ein noch ungelöstes Problem. Jedoch kann aus biochemischen und pharmakologischen Studien geschlossen werden, daß in dem vielstufigen Prozeß der biologischen Gedächtnisbildung ein physikalischer Teilschritt (plausibel) ist, der auf der Richtungsabhängigkeit von $\mathrm{Hy}$ sterese in Makromolekülen oder Membranen beruht ${ }^{[11]}$. Als weiterer dynamischer Aspekt von Hysterese bietet das Umlaufen der Hystereseschleife im Prinzip eine Möglichkeit, auf molekularer Ebene über Chemodiffusion periodische Vorgänge $z u$ erzeugen ${ }^{[10,12]}$. Diese Erkenntnis ist nicht nur für die Dynamik der Enzymkatalyse, sondern auch für die Versuche, biologische Rhythmen ${ }^{\left[1^{3]}\right.}$ auf oszillatorische chemische Reaktionen zurückzuführen, von groBem Interesse.

Besonders herausfordernd ist die Beobachtung von Hysterese in Nervenmembranen ${ }^{[14.15]}$. Es besteht kein Zweifel über die fundamentale Rolle elektrochemischer Erregbarkeit von Membranen für Kommunikation und Regelung in allen lebenden Organismen. Jedoch werden die Elementarprozesse der Erzeugung und Leitung bioelektrischer Impulse noch wenig verstanden. Es ist bemerkenswert, daß nach Adams molekularer Theorie der Nervenerregung die Auslösung der Aktionspotentiale über (kurzlebige) metastabile Zustände der Nervenmembran erfolgt ${ }^{[16]}$.

Aus diesen kurzen Hinweisen geht hervor, daß sich das Studium von Metastabilität und molekularer Hysterese, wie sie bei Makromolekülen und Membranen in Lösung und Suspension beobachtet wird, in die Bemühungen einordnen läßt, einige „biologische Erscheinungen auf physikalisch-mechanistische Grundkonzepte zurückzuführen" ${ }^{\text {"9]. }}$

\section{Thermodynamik der Hysterese}

\subsection{Hysterese und Metastabilität}

Um die speziellen dynamischen Eigenschaften von Hysterese zu verstehen, müssen wir tiefer in die Natur metastabiler Zustände eindringen. Wo immer langlebige Hysteresen auftreten, zeigen sie thermodynamisch metastabile Zustände und kooperative Nichtgleichgewichtsuibergänge an. Wie 
die makroskopischen Hystereseerscheinungen in und an kondensierten Phasen, scheint auch molekulare Hysterese in Lösung oder Suspension an Domänstrukturen gebunden zu sein. Unter einer Domäne versteht man allgemein eine Struktureinheit oder einen Strukturbereich, dessen Elementareinheiten ihren Ordnungszustand kooperativ, d.h. als Gesamtheit verändern können. Kooperative Strukturänderungen in Biopolymeren und Membranen können thermodynamisch als "diffuse" Phasenumwandlungen klassifiziert und mit guter Näherung als Phasenübergänge 1. Ordnung behandelt werden ${ }^{117-201}$.

Phasenumwandlungen sind erfahrungsgemä $ß$ oft an die Überwindung von Energiebarrieren gebunden, welche Hemmungen der Keimbildung oder des Keimwachstums bewirken. Um nun Gleichgewichtsumwandlungen zu realisieren, müssen diese Nucleations- und/oder Propagationsbarrieren verkleinert werden, etwa durch Schaffung von Keimbildungszentren. Falls dies jedoch unter gegebenen Bedingungen nicht möglich ist, weicht das System in metastabile Zustandsbereiche aus. So sind z. B. Überhitzungsund Unterkühlungserscheinungen bei thermischen Phasenübergängen Manifestationen von Umwandlungsbarrieren.

Thermodynamisch kann das Umwandlungsverhalten kooperativer Systeme mit metastabilen Zuständen durch eine Zustandsfunktion $\mathrm{y}(\mathrm{x})$ vom van-der-Waals-Typ beschrieben werden ${ }^{[21]}$. Eine solche Funktion ist in Abbildung 2a für die Umwandlung einer Domäne zwischen zwei Zuständen I und II schematisch dargestellt. Zwischen den beiden stabilen Ästen dieser Zustandskurve ist ein (gestrichelt gezeichneter) nicht realisierbarer Instabilitätsbereich. An der Stelle $x(G)$ befindet sich zwischen B und D der Gleichgewichtsübergang $\mathrm{I} \rightleftharpoons \mathrm{II}$.

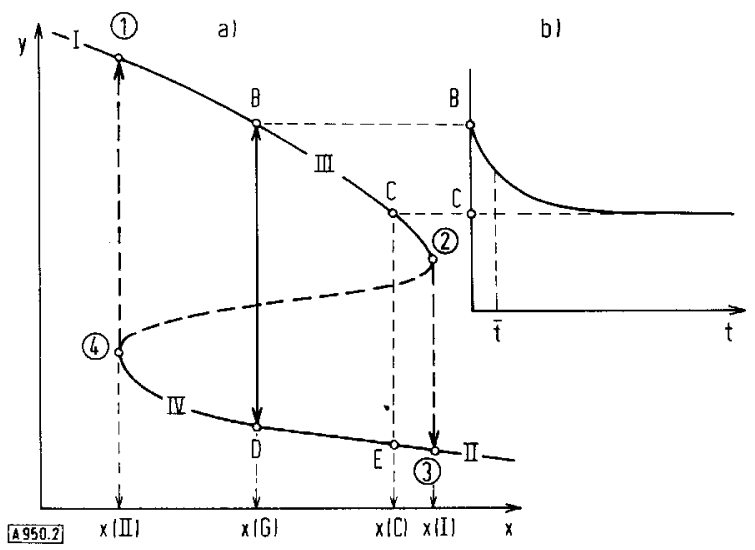

Abb. 2. a) Schematische Darstellung einer Phasenumwandlung. I, II stabile Zustände; III, IV: metastabile Zustände. y: interner Parameter, z. B. Volumen oder Reaktionsausmaß; x : externer Parameter, z. B. Temperatur oder $\mathrm{pH}-$ Wert. Vgl. Text. b) Zeitverlauf $\mathrm{y}(\mathbf{t})$ nach einer Änderung $\mathrm{x}(\mathrm{G}) \rightarrow \mathrm{x}(\mathrm{C})$; siehe Text.

Wenn nun genügend hohe Energiebarrieren eine Gleichgewichtsumwandlung verhindern, können Zustandsänderungen nur nach Passieren metastabiler Bereiche stattfinden. Zustand I ist metastabil zwischen B und 2, Zustand II ist metastabil zwischen D und 4. Die tatsächlichen Phasenübergänge sind einseitig gerichtet und irreversibel; sie erfolgen bei verschiedenen Werten für $\mathrm{x}$. Bei $\mathrm{x}(\mathrm{I})$ ist der Übergang von I nach II (zwischen 2 und 3); der Übergang von II nach I (zwischen 4 und 1) erfolgt jedoch bei $x$ (II). Damit legen die irreversiblen Phasenübergänge eine Umlaufrichtung für die Hysterese fest: $1 \rightarrow 2 \rightarrow 3 \rightarrow 4 \rightarrow 1$.

Das Auftreten von Hysterese ist nicht an die Bedingung von Metastabilität für beide Zustände I und II geknüpft. Es ist ausreichend, wenn nur ein Zustand Metastabilität entwickeln kann, z. B. Zustand II im Zustandsbereich IV. Für diesen Fall ist der Übergang IV nach I irreversibel, und die Umwandlung von I nach II vollzieht sich auf dem Gleichgewichtsweg bei $x(G)$.

Wie aus Abbildung 2 ersichtlich, ist im Hysteresebereich zwischen den Umwandlungspunkten der interne Parameter y keine ein-eindeutige Funktion der äußeren Variablen $\mathrm{x}$. Im Gegensatz zu Gleichgewichtskurven sind bei Hysterese jedem Wert von $\mathrm{x}$ zwei Werte von y zugeordnet. Je nach Ausgangszustand, I oder II, und Richtung der Änderung von $\mathrm{x}$, befinden wir uns entweder auf dem oberen oder unteren Ast der Zustandsfunktion. Das System ,erinnert" sich im Hysteresebereich an seine Vorgeschichte, an die Richtung des Weges, auf dem die Zustandsänderung erreicht wurde. Es ist nun diese einfache Form des Weggedächtnisses, das Hysterese von einem Gleichgewicht unterscheidet. Danach ist ein Gleichgewichtszustand ,gedächtnislos". Wohl kann Gleichgewicht auf verschiedenen Wegen erreicht werden; hat sich der Gleichgewichtszustand jedoch einmal eingestellt, „vergil $3 \mathrm{t}^{\text {" }}$ das System vorhergegangene Veränderungen. Der gedächtnislose Zustand des Gleichgewichts ist somit das tote Ende aller wegabhängigen, irreversiblen Prozesse ${ }^{[9]}$

Abrupte, irreversible Übergänge sind charakteristisch für das Umwandlungsverhalten einer einzelnen Domäne. In einem System von Domänen unterschiedlicher Stabilität führt die endliche Verteilung der Umwandlungspunkte zu scheinbar glatten Hysteresekurven, in denen die aufeinanderfolgenden irreversiblen Übergänge individueller Domänen innerhalb der Meßgenauigkeit nicht mehr unterscheidbar sind. Strukturuntereinheiten unterschiedlicher Größe und Stabilität sind Ursache für das Auftreten von ScanningKurven. Während Hysterese Indikator für Kooperativität und Metastabilität ist, deuten Scanning-Kurven das Vorliegen von „Polykristallinität“ oder Domänstrukturen an. So klassifiziert die Beobachtung von Scanning-Kurven das rRNA-Molekül als ein solches ,polykristallines“ Multidomänsystem.

\section{2. ,Zeitunabhängigkeit" stabiler Hysterese}

Wie schon erwähnt, wird Hysterese thermodynamisch metastabilen Zuständen zugeschrieben ${ }^{[22]}$, deren Lebensdauer jedoch von einem System zu einem anderen verschieden sein kann.

In Abbildung $2 \mathrm{a}$ und $2 \mathrm{~b}$ ist skizziert, wie eine Hysteresedomäne auf eine Änderung der Umgebung reagieren kann. Das System befinde sich z. B. im Zustand I bei Punkt $B$ auf der Zustandskurve und werde von $x(G)$ nach $x(C)$ ausgelenkt. In typischen Fällen wird nun beobachtet, daß 
sich der interne Meßparameter nach anfänglich schneller Änderung (mit der Halbwertzeit $\overline{\mathrm{t}}$ ) auf einen scheinbar konstanten Wert $\mathrm{C}$ einstellt. Bleibt dieser Wert für lange Beobachtungszeiten $t \gg \bar{t}$ und im Rahmen der Meßgenauigkeit unverändert, können wir der zugrundeliegenden Metastabilität auf dieser relativen Zeitbasis Langlebigkeit zuordnen. Gilt die scheinbare zeitliche Unveränderlichkeit nur für kürzere Beobachtungszeiten, so spricht man von kurzlebigen metastabilen Zuständen.

Für den Grenzfall, daß die Übergangswahrscheinlichkeit für eine Umwandlung von $\mathrm{C}$ zum Gleichgewicht $\mathrm{E}$ extrem klein wird, bleibt der Bruttoumsatz $\xi$ trotz endlicher Affinität A scheinbar konstant, d.h. $\frac{\mathrm{d} \xi}{\mathrm{dt}}=0$. Der Domänzustand $\mathrm{C}$ ist damit vergleichbar mit einem Zustand gehemmten Gleichgewichts $\left(A>0 ; \frac{d \xi}{d t}=0\right)$, wie ihn beispielsweise die bei Raumtemperatur (metastabile) Mischung von $\mathrm{H}_{2}$ und $\mathrm{O}_{2}$ gegenüber $\mathrm{H}_{2} \mathrm{O}$-Dampf darstellt.

Die scheinbare Zeitunabhängigkeit gestattet es, für metastabile Zustände thermodynamische Zustandsgrößen in gleicher Weise zu verwenden wie für Gleichgewichtszustände.

Das Zustandsverhalten einer Domäne ist scheinbar reversibel, wenn die Auslenkungen auf die Metastabilitätsbereiche beschränkt bleiben. Wird in unserem Beispiel die Auslenkung von $x(G)$ nach $x(C)$ wieder rückgängig gemacht, kehrt die Domäne in den Zustand $\mathrm{B}$ zurück. Wenn jedoch die Umweltänderung über $x(\mathrm{I})$ hinausgeht, so daß die irreversible Umwandlung nach II ablaufen kann, bleibt die hervorgerufene Zustandsänderung wegen der Gerichtetheit des Übergangs $2 \rightarrow 3$ auch bei Rückkehr zum Ausgangswert $x(G)$ bestehen. Erst eine Änderung von $x$ in die ent gegengesetzte Richtung über $x$ (II) hinaus kann die Domäne wieder in ihren ursprünglichen Zustand B zurückbringen. Dieses Nichtgleichgewichtsverhalten stempelt eine Domäne zu einer Matrize für eine bleibende Aufprägung gerichteter chemischer oder physikalischer Änderungen der Umgebung.

Erstreckt sich eine zyklische Änderung von $x$ über beide Übergangspunkte $x(I)$ und $x(I I)$ hinaus, hängt im allgemeinen die Größe der Hystereseschleife von der Zyklisierungsgeschwindigkeit ab. Kurzlebige Metastabilität führt zu den Relaxationshysteresen, die im Grenzfall langsamen Umlaufs zur Gleichgewichtskurve „zusammenschrumpfen“. Stabile Hysteresen ändern hingegen ihre Größe bei Verringerung der Umlaufgeschwindigkeit (im Rahmen der Zeitunabhängigkeit der zugrundeliegenden langlebigen metastabilen Zustände) nicht.

\subsection{Entropieproduktion}

Die Richtung des Umlaufs einer Hysterese ist durch den Entropieanstieg festgelegt, welcher mit gerichteten (irreversiblen) Zustandsänderungen einhergeht. Die Entropieänderung dS eines solchen Reaktionssystems ist (als Produkt mit der absoluten Temperatur T) mit der Änderung der inneren Energie d U, des Arbeitsterms dW und des chemischen Anteils $A \cdot d \xi$ in der Gibbs-Gleichung für irreversibel ablaufende chemische Reaktionen (oder Phasenänderungen) korreliert ${ }^{[23]}$ :

$\mathrm{TdS}=\mathrm{dU}+\mathrm{dW}+\mathbf{A d} \xi$

In den Definitionsgleichungen für die Affinität und für die Änderung des Reaktionsausmaßes

$A=-\sum_{j} v_{j} \mu_{j} ; \quad d \xi=v_{j} d n_{i}$

ist $\mu_{\mathrm{j}}$ das chemische Potential, $v_{\mathrm{j}}$ der stöchiometrische Koeffizient und $\mathrm{n}_{j}$ die Molzahl der $\mathrm{j}$-ten Komponente (oder einer Komponente in der j-ten Phase).

In der Nichtgleichgewichtsthermodynamik ist es üblich, die Entropieänderung eines Reaktionssystems in die zwei Terme $d_{e} S$ und $d_{i} S$ aufzuteilen ${ }^{[24]}$, so $d a \beta$

$\mathrm{dS}=\mathrm{d}_{\mathrm{e}} \mathrm{S}+\mathrm{d}_{\mathrm{i}} \mathrm{S}$

Der Term $d_{e} S$ ist der mit der Umgebung (als Wärme dQ) austauschbare Anteil, $d_{e} S=d_{e} Q / T$, während $d_{i} S$ den im System intern erzeugten, irreversiblen Anteil der Entropie bedeutet. Durch Definition ist $\mathrm{d}_{\mathrm{i}} \mathrm{S}$ positiv, $\mathrm{d}_{\mathrm{i}} \mathrm{S} \geq 0$. Benutzen wir nun den 1 . Hauptsatz, $d U=d_{e} Q-d W$, und setzen Gl. (3) in Gl. (1) ein, so erhalten wir

$\mathrm{Td}_{\mathrm{i}} \mathrm{S}=\mathrm{A} \cdot \mathrm{d} \xi$

In dieser Beziehung ist ausgedrückt, daß in einem Reaktionssystem Entropieerzeugung $\left(\mathrm{d}_{i} \mathrm{~S}>0\right)$ nur dann auftritt, wenn die betreffende Reaktion oder Phasenänderung irreversibel abläuft (d.h. wenn $A>0$ ist).

Die Entropieproduktion für einen Hysteresezyklus $\Delta_{\mathrm{i}} \mathrm{S}$ ergibt sich durch zyklische Integration von Gl. (4) in allgemeiner Form:

$\Delta_{\mathrm{i}} \mathrm{S}=\oint \mathrm{d}_{\mathrm{i}} \mathrm{S}=\oint \frac{\mathrm{A}}{\mathrm{T}} \mathrm{d} \xi$

Wie erwähnt, bietet die der Hysterese innewohnende (intrinsische) Richtungsabhängigkeit eine Möglichkeit, „Information" aufzuzeichnen (und durch Fortführen des Zyklus wiederum zu löschen). Diese Information (= Negentropie) basiert auf der Entropieproduktion der Aufzeichnungsmatrize. Speicherung wie Löschung des „Aufdrucks“ sind jedoch durch Arbeitsaufwand „erkauft“, denn Entropieerzeugung bedeutet Verlust brauchbarer Arbeit. Dieser Sachverhalt geht unmittelbar aus Gl. (1) hervor. Für einen geschlossenen Umlauf gilt

$T \oint \mathrm{d} S=\oint \mathrm{d} U+\oint \mathrm{d} W+\oint \mathrm{Ad} \xi$

Da $S$ und $U$ nur vom Zustand des Systems abhängen, sind (für eine zum Ausgangszustand zurückkehrende Änderung) die zyklischen Integrale $\oint \mathrm{dU}$ und $\oint \mathrm{dS}$ gleich Null. Damit reduziert sich Gl. (6) auf die Beziehung

$-\oint \mathrm{d} W=\oint \operatorname{Ad} \xi$ 
und mit Gl. (5) erhalten wir:

$\Delta_{\mathrm{i}} \mathrm{S}=-\frac{1}{\mathrm{~T}} \oint \mathrm{dW}$

Für $\Delta_{\mathrm{i}} \mathrm{S}>0$ folgt $\oint \mathrm{dW}<0$; d.h., um einen irreversiblen Zyklus zu schließen, muß Arbeit investiert werden.

\subsubsection{Isotherm-isobare Hysterese}

Für einen chemischen Prozeß, der bei konstanter Temperatur . T und konstantem Druck p irreversibel abläuft, ist die Änderung der Freien Enthalpie dG durch

$(\mathrm{dG})_{\mathrm{q}, \mathrm{T}}=-\mathrm{Ad} \xi<0$

gegeben. $\mathrm{Zu}$ Vergleichszwecken bezeichnen wir nun das Produkt $-\mathrm{T}\left(\Delta_{\mathrm{i}} \mathrm{S}\right)_{\mathrm{p} . \mathrm{T}}$ als $\left(\Delta_{\mathrm{i}} \mathrm{G}\right)_{\mathrm{p} . \mathrm{T}}$; das ist der Betrag der Freien Enthalpie, der bei einem isotherm-isobaren Hystereseumlauf ,verloren“ geht.

$\left(\Delta_{i} G\right)_{p \cdot I}=-T \cdot\left(\Delta_{i} S\right)_{p, r}$

Diese Energiedissipation resultiert aus allen im System irreversibel ablaufenden Prozessen k. Durch Vergleich von Gl. (5) und Gl. (10) erhalten wir

$\left(\Delta_{i} G\right)_{p, T}=-\sum_{k} \int_{0}^{\xi_{k}} A_{k} d \xi_{k}$

In G1. (11) ist der Umsatzparameter normiert; $\xi$ variiert als relatives Reaktionsmaß oder Umwandlungsgrad zwischen $\xi=0$ und $\xi=1$.

\subsubsection{Berechnung von $\Delta \mathrm{G}$}

Die Berechnung von $\Delta_{i} \mathrm{G}$ läßt sich in allgemeiner Form mit den in Abbildung 2a definierten Zustandssymbolen nach dem Schema von Abbildung 3 durchführen ${ }^{[25]}$.

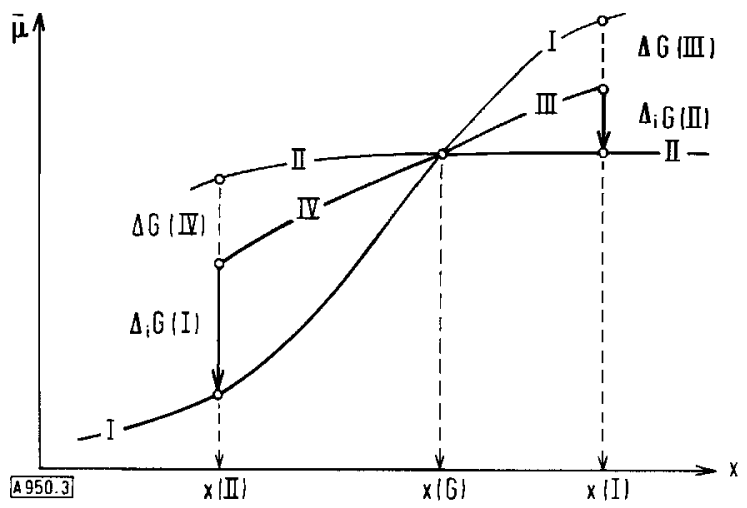

Abb. 3. Projektion der mittleren chemischen Potentiale $\bar{\mu}$, die den Reaktionskomponenten (oder Zuständen) zugeord net sind, in eine $\bar{\mu}-\mathrm{x}$-Ebene. Vgl. dazu Text und Abb. 2a.

Werden z. B. die Zustände I und II als Bezugszustände gewählt, ist der Unterschied der Freien Enthalpie $\Delta \mathrm{G}$ (II) zwischen den Zuständen II und I an der Stelle $\mathrm{x}$ (II) formal gleich der Summe der Energieänderung $\Delta G(I V)$, für den Übergang II nach IV bei $x$ (II), und des irreversiblen Anteils $\Delta_{\mathrm{i}} \mathrm{G}(\mathrm{I})$.

$\Delta \mathrm{G}(\mathrm{II})=\Delta \mathrm{G}(\mathrm{IV})+\Delta_{\mathrm{i}} \mathrm{G}(\mathrm{I})$
Entsprechend gilt bei $\mathrm{x}(\mathrm{I})$ :

$\Delta G(I)=\Delta G(I I I)+\Delta_{i} G(I I)$

Da $\Delta_{\mathrm{i}} \mathrm{G}=\Delta_{\mathrm{i}} \mathrm{G}(\mathrm{II})+\Delta_{\mathrm{i}} \mathrm{G}(\mathrm{l})$ ist, folgt für den Gesamtwert der Dissipation an Freier Enthalpie bei einem Hystereseumlauf die Beziehung

$\Delta_{i} G=\Delta G(I)+\Delta G(I)-\Delta G(I V)-\Delta G(I I I)$

\subsubsection{Thermische (isobare) Hysterese}

Wenn bei thermischer Hysterese der Umwandlungsgrad $\xi$ als Funktion der Temperatur bei konstantem Druck bestimmt werden kann, läßt sich aus Gl. (5) eine Beziehung zur Messung der Entropieproduktion $\left(\Delta_{i} S\right)_{p}$ ableiten.

$\left(\Delta_{i} S\right)_{p}=\oint\left[\sum_{i} \frac{A_{k}}{T}\left(\frac{d \xi_{k}}{d T}\right)\right] d T$

\subsection{Energiedissipation bei potentiometrischen Hysteresen}

Die Hystereseschleifen, die bei isotherm-isobaren potentiometrischen Säure-Base-Titrationen erhalten werden, sind ein direktes Maß für die Energiedissipation und Entropieproduktion ${ }^{[25]}$. Bei der potentiometrischen Hysterese (vgl. z. B. Abb. 4) entspricht der mittlere Protonierungsgrad $\bar{\alpha}$ dem internen Parameter y und der $\mathrm{pH}$-Wert der externen Variablen $\mathrm{x}$ (in der Bezeichnungsweise von Abb. 2). Der Protonierungsgrad ist als das Verhältnis zwischen der Molzahl gebundener Protonen und der Molzahl der Protonierungspartner definiert.

Die übliche Bedingung einer solchen Säure-Base-Titration ist die Beibehaltung konstanter Ionenstärke während des Titrationszyklus, z. B. durch ein Neutralsalz, dessen Konzentration groß gegenüber den Konzentrationen der Reaktionspartner ist. Wenn zusätzlich Säure-Anion und SalzAnion identisch, beispielsweise $\mathrm{Cl}^{-}$-Ionen sind, so daß das chemische Potential dieser Anionen als konstant betrachtet werden kann, folgt z. B. für die Kombination $\mathrm{HCl}$ und $\mathrm{NaCl}: \mathrm{d} \mu_{\mathrm{HCl}}=\mathrm{d} \mu_{\mathrm{II}^{+}}+\mathrm{d} \mu_{\mathrm{Cl}^{-}} \simeq \mathrm{d} \mu_{\mathrm{II}-}$. Unter dieser Voraussetzung ist der Anteil der Protonierung an der Änderung des chemischen Potentials des (protonierten) Reaktionspartners $\mathrm{j}$ mit dessen Protonierungsgrad $\alpha_{\mathrm{j}}$ und dem $\mathrm{pH}$ Wert in guter Näherung durch die Beziehung

$\left(\frac{\partial \mu_{j}}{\partial p H}\right)_{n \neq n_{H C I}}=-2.3 R T \alpha_{j}$

verknüpft. Die Bezeichnung $n \neq n_{\mathrm{FICl}}$ bedeutet konstante

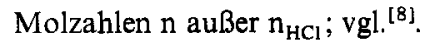

Der Anteil der Komponente $\mathrm{j}$ an der Änderung der Freien Enthalpie $\Delta \mathrm{G}_{\mathrm{k}}$ der k-ten Protonierungsreaktion bei einem Säure-Base-Zyklus kann durch zyklische Integration von Gl. (14) abgeleitet werden.

$\Delta G_{k}=\oint\left(\begin{array}{c}\partial \mu_{j} \\ \partial p H\end{array}\right)_{k} \mathrm{dpH}=-2.3 \mathrm{RT} \oint \alpha_{j \mathrm{k}} \mathrm{dpH}$

Da durch Definition

$\Delta G_{k}=-\int_{0}^{\check{\zeta}_{k}} A_{k} d \check{\zeta}_{k} ; \quad\left(\Delta_{i} G\right)_{p, T}=\sum_{k} \Delta G_{k}$ 

gilt, ergibt sich mit Gl. (11) und mit $\bar{\alpha}=\sum_{\mathbf{j}} \sum_{\mathbf{k}} \alpha_{\mathbf{j k}}$ für die
Gesamtdissipation an Freier Enthalpie:

$\left(\Delta_{\mathrm{i}} \mathrm{G}\right)_{\mathrm{p} . \mathrm{T}}=-2.3 \mathrm{RT} \oint \bar{x} \mathrm{dpH}$

Das Integral $\oint \bar{\alpha} \mathrm{dpH}$ ist geometrisch die Fläche der potentiometrischen Hystereseschleife. Damit sind $\left(\Delta_{\mathrm{i}} \mathrm{G}\right)_{\mathrm{p}, \mathrm{T}}$ und die Entropieproduktion

$\left(\Delta_{\mathrm{i}} \mathrm{S}\right)_{\mathrm{p} . \mathrm{T}}=2.3 \mathrm{~T} \oint \overline{\mathrm{x}} \mathrm{dpH}$

experimentell bestimmbar.

Berechnung von $\bar{\alpha}$. Die Anzahl gebundener Protonen kann aus dem. Unterschied der Mengen an Säure oder Base errechnet werden, die notwendig sind, um die Lösung oder Suspension der Protonierungspartner auf den gleichen $\mathrm{pH}$ Wert zu bringen wie eine Vergleichslösung ohne die betreffende Reaktionskomponente. Da die Untersuchung von Hystereseerscheinungen mehr als einen Säure-Base-Zyklus erfordert, müssen ausführlichere Beziehungen zur Berechnung von $\bar{\alpha}$ benutzt werden als sonst üblich ist. Solche allgemeinen Gleichungen sind von Revzin, Neumann und Katchalsky angegeben worden ${ }^{[7]}$.

\subsection{Protonierungsenergie polyelektrolytischer Systeme}

In den Bereichen der Hysterese, in denen die Protonierung reversibel oder scheinbar reversibel (Metastabilitätsbereiche) ist, läßt sich der Formalismus des Protonierungsgleichgewichts anwenden. Bei polyelektrolytischen Systemen ist die $\mathrm{pH}$-Abhängigkeit des Protonierungsgrads $\alpha_{\mathrm{j}}$ der Molekülgruppe $\mathrm{j}$ durch die Beziehung

$\log \frac{1-x_{j}}{x_{j}}=p H-p K_{j}^{0}-\Delta p K_{j}$

gegeben. In Gl. (19) ist $\mathrm{pK}_{\mathrm{j}}^{0}$ der $\mathrm{pK}$-Wert des einzelnen Monomerbausteins $\mathrm{j}$, und der Polyelektrolyt-Anteil der pK-Verschiebung ist durch die Gleichung

$\Delta \mathrm{pK}_{\mathbf{j}}=\frac{\mathrm{z}_{\mathbf{j}} \cdot \mathrm{e} \cdot \psi_{\mathbf{j}}}{2.3 \mathrm{kT}}$

definiert ${ }^{\{26 \mid}$. In Gl. (20) ist e die (positive) Elementarladung, $\mathrm{k}$ die Boltzmann-Konstante, $\psi_{\mathrm{j}}$ das elektrische Potential an der Stelle der Protonierung $\mathbf{j}$ und $z_{j}$ die (negative oder positive) Ladungszahl der das Potential $\psi_{\mathrm{j}}$ bestimmenden Gruppen. Der Wert $\psi_{\mathrm{j}}$ ist abhängig von der Geometrie des Systems, dem mittleren Protonierungsgrad und der Ionenstärke des Mediums.

Für die thermodynamische Analyse potentiometrischer Hysteresen ist es oft erforderlich, die Freie Enthalpie der Protonierung $\Delta_{\mathrm{p}} \mathrm{G}$ zu berechnen. Der Standardwert für die Gruppe $\mathrm{j}$ ist

$\Delta_{r} G_{j}^{0}=-2.3 R T\left(\mathrm{pK}_{j}^{0}+\Delta \mathrm{pK}_{\mathrm{j}}\right)$
Bei Kenntnis des Monomer-pK-Werts reduziert sich die $\Lambda_{\mathrm{p}} \mathrm{G}_{\mathrm{j}}^{0}$-Bestimmung auf die Berechnung von $\Delta_{\mathrm{p}} \mathrm{K}_{\mathrm{j}}$; vgl. dazul ${ }^{20 !}$.

\subsection{Ziel thermodynamischer Hystereseanalyse}

Die in Abschnitt 2 diskutierten thermodynamischen Beziehungen stellen den allgemeinen Rahmen für die Analyse von Hystereseerscheinungen bei chemischen Reaktionen oder Phasenänderungen dar. Die zentrale Aufgabe einer solchen Analyse besteht in der Identifizierung derjenigen Prozesse, die irreversibel ablaufen. Dazu kann der Vergleich der gemessenen und berechneten Werte für die Energiedissipation $\Delta_{\mathrm{i}} \mathrm{G}$ benutzt werden.

Besonders bei polyelektrolytischen Systemen sollte die thermodynamische Analyse von Temperatur- und Ionenstärkeabhängigkeiten der Hysterese auch Hinweise auf die Natur der Energiebarriere geben, welche die metastabilen Zustände bedingt und das System zum hysteretischen Umgehen dieser Barriere zwingt.

Aus einem Vergleich der Beziehungen (8) und (10) geht hervor, daß $\left(\Delta_{\mathrm{i}} G\right)_{p . T}$ ein Maß für den Energieaufwand bei einem isotherm-isobaren Hystereseumlauf ist. Daher führt die thermodynamische Analyse im Prinzip zu der Bestimmung der Freien Enthalpien, welche dem System zur Wiederherstellung der metastabilen Zustände zugeführt werden müssen. Die Rolle metastabiler Zustände bei der Auslösung gerichteter Prozesse unter geringem Energieaufwand wird in den Abschnitten 7 und 8 erörtert.

\section{Hysterese bei Nucleinsäuren}

\subsection{Synthetische Polynucleotide}

Besonders ausgeprägte Hystereseerscheinungen werden bei potentiometrischen und spektrophotometrischen Titrationen von Komplexen homogener synthetischer Nucleinsäuren beobachtet. Als repräsentatives Beispiel ist in Abbildung 4 das Ergebnis einer potentiometrischen Säure-BaseTitration des Polynucleotidkomplexes Poly(A) 2 Poly(U) dargestellt ${ }^{[8]}$.

Dieser dreistrangige Komplex entsteht bei der Mischung von Polyriboadenylat, Poly(A), und Polyribouridylat, Poly(U), im Molverhältnis der Polymeren 1:2 bei neutralem $\mathrm{pH}$ und genügend hoher Ionenstärke ${ }^{[27]}$. Der zweistrangige Komplex Poly(A). Poly(U) kann durch Mischung der Einzelstränge im Molverhältnis $1: 1$ erhalten werden ${ }^{[28]}$. In diesen helicalen Mehrstrangmolekülen sind die heterocyclischen Basen Adenin und Uracil über $\mathrm{H}$-Brückenbindungen spezifisch zugeordnet und regelmäßig aufeinandergestapelt. Aufgrund der kooperativ stabilisierten Basenstapelung können solche Systeme als ,eindimensionale“ Kristalle betrachtet werden. (Die Stabilität mehrsträngiger Polynucleotidkomplexe ist wegen der ausgeprägten Polyelektrolytnatur aller Nucleinsäuren relativ stark von der Ionenstärke des Mediums abhängig.)

Die ersten Anzeichen für metastabile Formen im A-U-System tauchen bei Befunden von Warner und Breslow auf: 
$\operatorname{der}(\mathrm{A} \cdot \mathrm{U})$-Komplex, der bei $\mathrm{pH}=5.5$ gegenüber den Einzelsträngen scheinbar stabil ist, bildet sich jedoch nur in geringem Ausmaß, wenn die Polymeren getrennt erst auf pH 5.5 gebracht und dann gemischt werden ${ }^{[28]}$. Ähnliche Ergebnisse erhielten Steiner und Beers ${ }^{[29]}$. Cox entdeckte anschließend, daß bei spektrophotometrischen Titrationen von Lösungen des (A.U)-Komplexes Hystereseschleifen auftreten, deren Größe von der Temperatur abhängig ist $^{[30]}$.

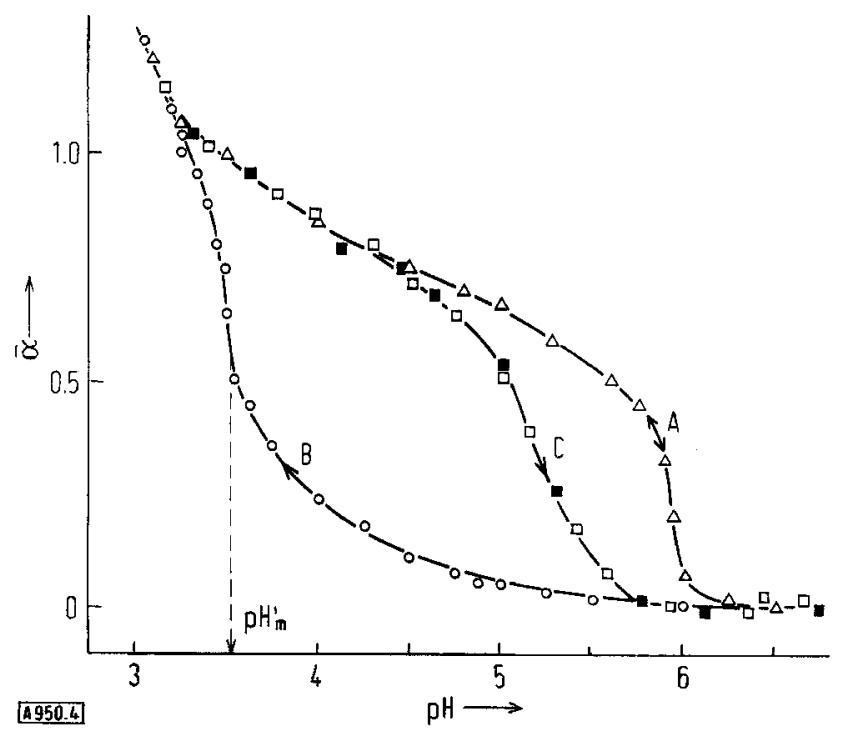

Abb. 4. Potentiometrische Titrationen (Protonierungsgrad $\bar{\alpha}$ als Funktion des $\mathrm{pH}$-Werts) bei $20^{\circ} \mathrm{C}$ : a) einer $0.1 \mathrm{M} \mathrm{NaCl}-$ Lösung von Poly(A) und Poly(U), gemischt im Molverhältnis der Polymeren von 1:2, 0 : Säuretitration (Kurve $B$ ), 0 : anschlie(Bende Basetitration (Kurve C); - b) einer $0.1 \mathrm{M} \mathrm{NaCl}$-Lösung von Poly(A), $\Delta$ (Kurve A). Polymerkonzentrationen bei Titrationsbeginn, $\mathrm{pH} 7:$ a) $\mathrm{c}=3.24 \cdot 10^{-4} \mathrm{M}(\mathrm{A} \cdot 2 \mathrm{U})$; b) $\mathrm{c}=$ $3.2 \cdot 10^{-4} \mathrm{M}(\mathrm{A})$

Auch Poly(A) zeigt Abhängigkeit von der „Vorgeschich-

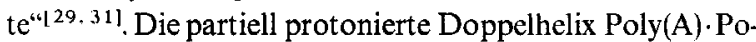
ly(A), die dieses Polymer bei saurem $\mathrm{pH}$ zu bilden vermag, tendiert mit steigender Protonenkonzentration zur Aggregation. Das Ausmaß der Aggregatbildung ist bei konstantem $\mathrm{pH}$ von der Ionenstärke abhängig. Der Zustand des Systems hängt nun von der Reihenfolge $a b$, in der die Parameter $\mathrm{pH}-$ Wert und Ionenstärke geändert werden.

Die Aggregatbildung von Poly(A) erfolgt bei nicht zu niedrigem $\mathrm{pH}$ jedoch relativ langsam, so daß dadurch die experimentelle Untersuchung der Säure-Base-Hysterese im A-U-System nicht erheblich gestört wird. (Aggregation größeren Ausmaßes läßt sich durch Messung von Sedimentationskoeffizienten prüfen.)

\subsubsection{Das (A.2U)-Modellsystem}

Die ersten thermodynamischen Analysen molekularer $\mathrm{Hy}$ sterese sind von Katchalsky, Oplatka und Litan am A-U-Sysystem anhand der Coxschen Meßergebnisse durchgeführt worden ${ }^{[25]}$. Im Gegensatz zu der ursprünglichen Interpretation ist es jedoch weder die (A.U)-Doppelhelix noch $\operatorname{der}(\mathrm{A} \cdot \mathrm{A})-\mathrm{Komplex}$, sondern nur $\operatorname{der}(\mathrm{A} \cdot 2 \mathrm{U})$-Tripelstrang, der im A-U-System langlebige Metastabilität entwickeln $\mathrm{kann}^{[8]}$

Die Hysterese in der Säure-Base-Titration im A-U-System begleitet die wechselseitige „Umkristallisation“ zwischen
$\operatorname{der}(\mathrm{A} \cdot 2 \mathrm{U})$-Tripelhelix und dem (A.A)-Doppelstrang. Mit den Symbolen der reagierenden Kettenuntereinheiten oder Segmente lautet die Reaktion:

$2(\mathrm{~A} \cdot 2 \mathrm{U}) \underbrace{\stackrel{\leftrightarrow}{+}}(\mathrm{A} \cdot \mathrm{A})+4(\mathrm{U})$

Im $\mathrm{pH}$-Bereich der Titration ( $\mathrm{pH} 3$ bis 7) ist der in Abbildung 4 dargestellte Meßwert $\bar{\alpha}$ durch die Protonierung der (A)-Segmente bestimmt. Bei der Säuretitration geht $\operatorname{der}(\mathrm{A} \cdot 2 \mathrm{U})-\mathrm{Komplex}$ nicht direkt in die protonierte (A.A)Helix über, sondern wird zunächst selbst (partiell) protoniert. (Diese Annahme stïtzt sich auf die Ähnlichkeit der Sedimentationskoeffizienten-Verteilung zwischen $\mathrm{pH} 7$ und $\mathrm{pH}$ 4.) Die beiden Beiträge zum Meßwert $\bar{\alpha}$ sind demnach durch den Protonierungsgrad des (A.A)-Doppelstranges, $\alpha_{2}$, und durch den der Tripelhelix, $\alpha_{3}$, gegeben. Bezeichnen wir das ReaktionsausmaB der in G1. (22) beschriebenen Konformationsänderung mit $\xi$, so erhalten wir für jeden $\mathrm{pH}$-Wert:

$\bar{\alpha}=\xi \alpha_{2}+(1-\xi) \alpha_{3}$

Der Wert von $\alpha_{2}$ ist unabhängig durch Titration von Poly(A) bestimmbar, während $\alpha_{3}$ mit einer Gl. (19) analogen Beziehung berechnet werden kann; siehe Gl. (35).

In ähnlicher Weise können UV-spektrometrische Titrationen zur Ermittlung der pH-Abhängigkeit von $\xi$ verwendet werden. Abbildung 5 zeigt, daß dieselben $\xi(\mathrm{pH})$-Werte erhalten werden, unabhängig davon, ob die Beobachtungsgröße $\bar{\alpha}$ oder die UV-Absorption ist ${ }^{\left[{ }^{81}\right.}$. Es ist weiterhin ersichtlich, daß entlang des Säure-Asts die (A.A)-Bildung erst im Bereich um pH 3.5 einsetzt. Dieser Übergang ist abrupt, während der "glatte" Base-Ast auf eine Gleichgewichtsumwandlung hindeutet.

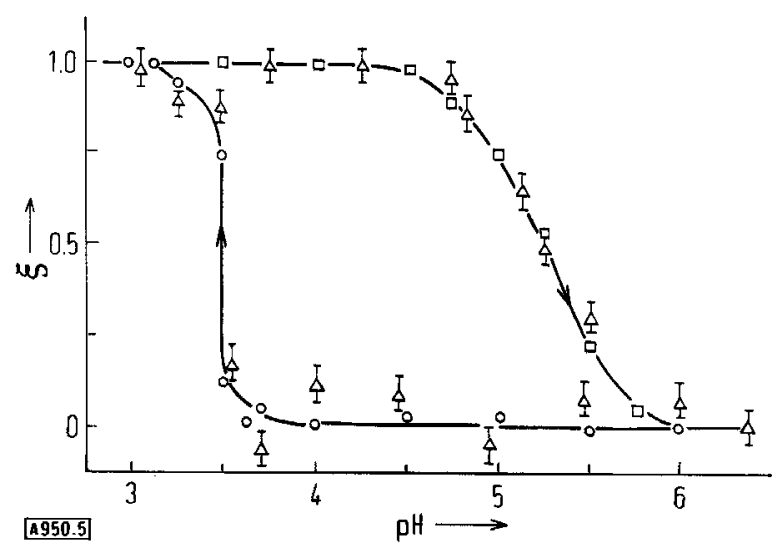

Abb. 5. Reaktionsausma $B$ als Funktion des $\mathbf{p H}$-Werts. Bedingungen wie in Abb. 4. $\circ, a$ : Daten aus potentiometrischer und $\triangle$ : Daten aus spektrophotometrischer Titration. Vgl. [8].

a) Molare Zustandsgrößen. Bei hochmolekularen Polynucleotiden sind die gemessenen (scheinbaren) Zustandsgrößen unabhängig von der Polymerkonzentration $c_{j}{ }^{[18,32]}$. Das berechtigt zur Annahme konstanter Aktivitätskoeffizienten und deren Einbeziehung in die Bezugspotentiale $\mu_{\mathrm{j}}^{0}$, so daß 
$\Delta G=\sum_{j} v_{j} \mu_{j}=\sum_{j} v_{j} \mu_{j}^{0}+R T \sum_{j} v_{j} \ln c_{j}$

ist. Für einen stöchiometrischen Umsatz, d.h. $c_{j}=1 \mathrm{~mol} / 1$, folgt

$\Delta G^{0}=\sum_{\mathbf{j}} v_{j} \mu_{j}^{0}$

und nach $\mathrm{Gl}$. (16) erhalten wir

$\left(\Delta_{\mathrm{i}} G\right)^{\theta}=\sum_{\mathrm{h}}\left(\sum_{j} v_{\mathrm{j} k} \mu_{\mathrm{j}}^{0}\right) \xi_{\mathrm{k}}$

Beziehen wir die Zustandsgrößen auf die Molzahl der (A)-Kettensegmente, ergibt sich die Freie Enthalpie, die bei einem isotherm-isobaren Hysteresezyklus dissipiert,

$\left(\Delta_{i} G\right)_{p-\mathrm{T}}^{0}=\sum_{k} \Delta G_{k}^{0} \cdot \zeta_{k}=-2.3 R T \oint \bar{x} \mathrm{dpH}$

pro mol (A), direkt aus der Meßkurve von Abbildung 4.

b) Berechnung von $\left(\Delta_{\mathrm{i}} G\right)^{0}$. Wie erwähnt, gibt es Hinweise darauf, daß der Base-Ast der Hysterese im A-U-System die Gleichgewichtskurve ist ${ }^{[8.33]}$. Demnach scheint unser Modellsystem nur einen Metastabilitätsbereich zu haben. In dem entsprechenden $\mathbf{p H}$-Intervall dieses Bereichs stellt die teilweise Protonierung der Tripelhelix ein metastabiles Vorgleichgewicht dar. Dieses Vorgleichgewicht umfaßt die Öfnung von (A-2 U)-Segmenten

$(\mathrm{A} \cdot 2 \mathrm{U}) \rightleftharpoons(\mathrm{A})+2(\mathrm{U})$

(28)

und die Protonierung von offenen (A)-Segmenten nach $(\mathrm{A})+\mathrm{H}^{+} \rightleftharpoons\left(\mathrm{AH}^{+}\right)$im Dreistrangkomplex.

Das Protonierungs-Vorgleichgewicht entspricht dem $\mathrm{Zu}$ stand IV in Abbildung 2a. Dem Zustand II ordnen wir demnach die (A.2U)-Tripelhelix und dem Zustand I die (A.A)-Doppelhelix zu. Werden nun die chemischen Potentiale der Einzelstränge als Bezugszustand gewählt, gestaltet sich die Berechnung von $\left(\Delta_{i} G\right)^{0}$ besonders einfach. Für unser Modellsystem reduziert sich die allgemeine Darstellung in Abbildung 3 auf das in Abbildung 6 gezeigte Sche$\mathrm{ma}^{[8]}$.

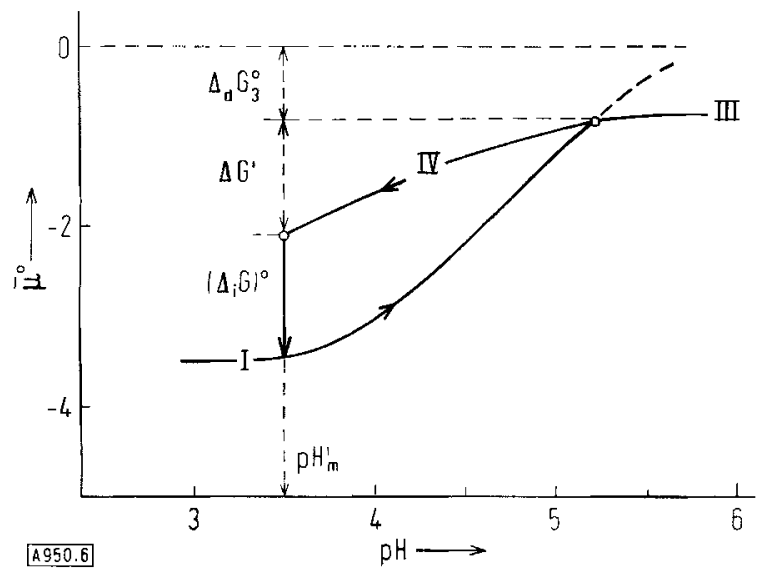

Abb. 6. Projektion der mittleren molaren Standardpotentiale $\bar{\mu}^{\circ}$, die den Nucleotidsegmenten zugeordnel sind. in die Potential-pH-Ebene: $\Delta\left(\mathrm{i}^{\prime}=\mathrm{x}_{3}^{\prime} \cdot\left(\Delta_{\mathrm{r} 1} \mathrm{G}_{3}^{\prime}+\Delta_{\mathrm{p}} \mathrm{G}_{3}^{()}\right)\right.$, siehe Gl. (29).
Die in diesem Fall der Gl. (12) entsprechende Beziehung erhalten wir mit Gl. (25) und (26):

$\left(\Delta_{i} G\right)^{0}=\Delta_{b} G_{2}^{0}+\Delta_{\mathrm{d}} G_{3}^{0}+\alpha_{3}^{\prime}\left(\Delta_{d} G_{3}^{0}+\Delta_{\mathrm{p}} G_{3}^{0}\right)$

In Gl. (29) ist $\Delta_{b} G_{2}^{0}$ die Änderung der Freien Enthalpie für die Reaktion $(\mathrm{A})+\mathrm{H}^{+}=\frac{1}{2}\left(\mathrm{AH}^{+} \cdot \mathrm{AH}^{+}\right)$bei $\mathrm{pH}_{\mathrm{m}}^{\prime}$ (entsprechend $x(I I)$ in Abb. 3); $\Delta_{d} G_{3}^{U}$ ist die Freie Enthalpie der Umwandlung nach Gl. (28); $\Delta_{\mathrm{p}} \mathrm{G}_{3}^{0}$ ist die Freie Enthalpie der Protonierung von (A)-Segmenten in der Tripelhelix (mit Gl. (21) berechenbar) und $\alpha_{3}^{\prime}$ ist das Ausmaß der Protonierung bei $\mathrm{pH}_{\mathrm{m}}^{\prime}$.

Im Rahmen der Annahmen, die Gl. (29) zugrundeliegen, stimmen Berechnung und Messung von $\left(\Delta_{\mathrm{i}} \mathrm{G}\right)^{0}$ überein ${ }^{[8]}$. Unter den experimentellen Bedingungen von Abbildung 4 findet man

$$
\begin{aligned}
& \left(\Delta_{\mathrm{i}} \mathrm{G}\right)_{\mathrm{p} . \mathrm{T}}^{0}=-1.4( \pm 0.1) \mathrm{kcal} / \mathrm{mol}(\mathrm{A}) \\
& \left(\Delta_{\mathrm{i}} \mathrm{S}\right)_{\mathrm{p} . \mathrm{T}}^{0}=4.9( \pm 0.1) \mathrm{cal} / \mathrm{mol}(\mathrm{A}) \mathrm{grad}
\end{aligned}
$$

Die Übereinstimmung von gemessenem und berechnetem $\Delta_{i} G$-Wert unterstützt die der Berechnung zugrundegelegte Interpretation des Hystereseverhaltens. Danach ist in unserem Modellsystem die Hysterese durch das metastabile Protonierungsgleichgewicht als Zwischenzustand bei der Säuretitration bedingt.

Es sei noch angefügt, daß der Energieverlust in diesem Hysteresesystem im Vergleich zur durchschnittlichen Stabilisierungsenergie chemischer Bindungen ( $\simeq 50 \mathrm{kcal} /$ mol) einen ziemlich kleinen Energieaufwand bedeutet.

\subsection{2. $(\mathrm{I} \cdot \mathrm{C})$ - und $(\mathrm{dC} \cdot \mathrm{dG})-$ Komplexe}

Dreisträngige Polynucleotidkomplexe scheinen besonders zur Ausbildung langlebiger Metastabilitäten zu tendieren. Polymerassoziate, die dem (A - 2U)-Komplex ähnlich sind, treten auch bei Komplexen von Polynucleotiden auf, deren Monomere Inosinsäure (I), Cytidyl (C)- und Guanylsäure (G) sind. Im System Poly(I) Poly(C) fanden Guschlbauer et al., daß in der $\mathrm{pH}$-A bhängigkeit des Circulardichroismus zwei Hysteresebereiche existieren, die durch einen Gleichgewichtsbereich voneinander getrennt sind $^{[34]}$. Als Zwischenzustand der (I.C)-Umwandlung kann (ähnlich wie bei der Umwandlung der (A.U)-Doppelhelix ${ }^{[8]}$ ) ein partiell protonierter Tripelstrang mit den Basenpaarungen (I C C C C ) identifiziert werden ${ }^{[35]}$,

Bei Polydesoxyribonucleotiden werden extrem ausgedehnte Metastabilitätsbereiche beobachtet. So erstreckt sich die Säure-Base-Hysterese in Poly $(\mathrm{dG}) \cdot \operatorname{Poly}(\mathrm{dC})$ über sechs pH-Einheiten (zwischen pH 2 und 8). Bei dieser Basenpaarung tritt im physiologisch relevanten $\mathrm{pH}$-Bereich um $\mathrm{pH}$ 7 Metastabilität auf. Vor dem Hintergrund längerer $(\mathrm{dC} \cdot \mathrm{dG})$-Bereiche in DNA ergibt sich hier ein Ansatzpunk1 für einen Regelmechanismus, der spezifisch auf Metastabilitäten in aufeinanderfolgenden $(\mathrm{dC} \cdot \mathrm{dG})$-Basenpaaren beruhen würde ${ }^{\mid 3+}$. Die Energieumsätze bei einem solchen Mechanismus könnten durch eine thermodynamische Analyse der $(\mathrm{dG} \cdot \mathrm{dC})$-Hysterese abgeschätzt werden. Im Prinzip kann eine derartige Analyse in ähnlicher Weise wie bcim (A.2U)-System durchgefuhrt werden. 


\subsubsection{Thermische Hysterese in Biopolymeren}

Bei einigen Komplesen aus Poly(dA) und Polymeren substituierter Uridylsäuren ändert sich die UV-Absorption während der Aufheizung und Abkühlung entlang einer Hystereseschleife ${ }^{[36]}$. Ähnliche thermische Hysteresen werden bei Thioderivaten des Copolymers Poly(dA-T) in Lösungen hoher Salzkonzentration $(2 \mathrm{M} \mathrm{NaCl})$ beobachtet ${ }^{[37]}$. Die Hysterese in diesem Copolymer geht mit Konformationsumwandlungen zwischen verschiedenen Helixtypen einher.

Die Fähigkeit zur Ausbildung metastabiler Zustände scheint nicht auf Komplexe aus mehreren Polyelektrolytsträngen beschränkt zu sein. Zum Beispiel zeigt der Komplex Poly(A). Poly(-I-vinyluracil), in dem nur Poly(A) ein Elektrolyt ist, thermische Hysterese über einen relativ weiten Temperaturbereich ${ }^{[38]}$.

Es gibt eine Reihe von Beispielen, bei denen Hysterese auf unspezifischer Aggregation und Desaggregation von Molekülen beruht. In diese Kategorie gehören wahrscheinlich die thermischen Hysteresen, die z. B. für eine Reihe von synthetischen Nucleinsäuren in Wasser-Methanol-Mischungen bei tieferen Temperaturen gefunden wurden ${ }^{[39]}$

Da optische Parameter wie die UV-Absorption bei bestimmten Wellenlängen ein MaB für den Umwandlungsgrad $\xi$ bei Konformationsänderungen sind, kann Beziehung (13) Ausgangspunkt für eine thermodynamische Beschreibung thermischer Hysterese in Biopolymeren sein, bei denen Strukturänderungen optisch verfolgt werden können.

\section{2. rRNA-Hysterese}

Nicht nur Mehrstrangkomplexe von Polynucleotiden sondern auch mehrstrangige kürzere Strukturbereiche in Nucleinsäuren können langlebige Metastabilitäten entwikkeln. Als repräsentatives Beispiel für diesen Fall gilt die Hysterese in rRNA; vgl. z. B $^{[40]}$. Neuere Studien an rRNA haben gezeigt, daß die rRNA-Hysterese intramolekularer Natur ist ${ }^{[7]}$. Die Ausbildung metastabiler Zustände ist bei diesem Biopolymer an die Anwesenheit von $\mathrm{Mg}^{2+}$-Ionen gebunden. Zudem treten Metastabilitäten nur entlang des Base-Asts der Grenzschleife und der Scanning-Kurven auf, während der Säure-Ast die Gleichgewichtskurve darstellt. Die hohe thermische Stabilität der metastabilen Zustände und die Analyse des UV-Spektrums als Funktion des $\mathrm{pH}$ Werts lassen darauf schließen, daß Strukturbereiche, die hauptsächlich aus protonierten $(\mathrm{G} \cdot \mathrm{G})$-Basenpaaren bestehen, der Grund für die langlebige Metastabilität in rRNA sind.

Die Frage nach der möglichen Rolle metastabiler Zustände in der rRNA-Struktur bei der Regelung von ribosomalen Prozessen ist Gegenstand weiterer Untersuchungen ${ }^{[7]}$.

\section{Hysterese bei Proteinen und Membranen}

Auch bei Strukturänderungen in ,globulären“ polyelektrolytischen Systemen wic Proteinen, Lipoprotein- und Lipid- vesikeln treten Hysteresen auf. Als repräsentatives Beispiel für Polypeptide gilt die Säure-Base-Hysterese der Polyglutaminsäure, die durch spezifische Aggregation-Desaggregations-Prozesse gedeutet wurde ${ }^{[41]}$.

Säure-Base-Titrationen werden bei Proteinen oft benutzt, um Art und Anzahl „,begrabener" Molekülgruppen zu ermitteln $^{[42]}$. Die in den Titrationskurven angezeigte Metastabilität ist jedoch selten thermodynamisch analysiert worden. Es sei darauf hingewiesen, daß die Energiedissipation $\Delta_{i} G$ bei solchen Systemen ein $M a ß$ für die Freie Stabilisierungsenergie der im Molekülinneren „begrabenen" Gruppen darstellt.

Ein Beispiel funktioneller Hysterese in Proteinen wurde in dem halophilen Enzym Malat-Dehydrogenase von Halobakterien aus dem Toten Meer entdeckt ${ }^{1431}$. Dieses Enzym ändert seine katalytische Aktivität als Funktion der $\mathrm{NaCl}$-Konzentration entlang einer Hystereseschleife (Abb. 7).

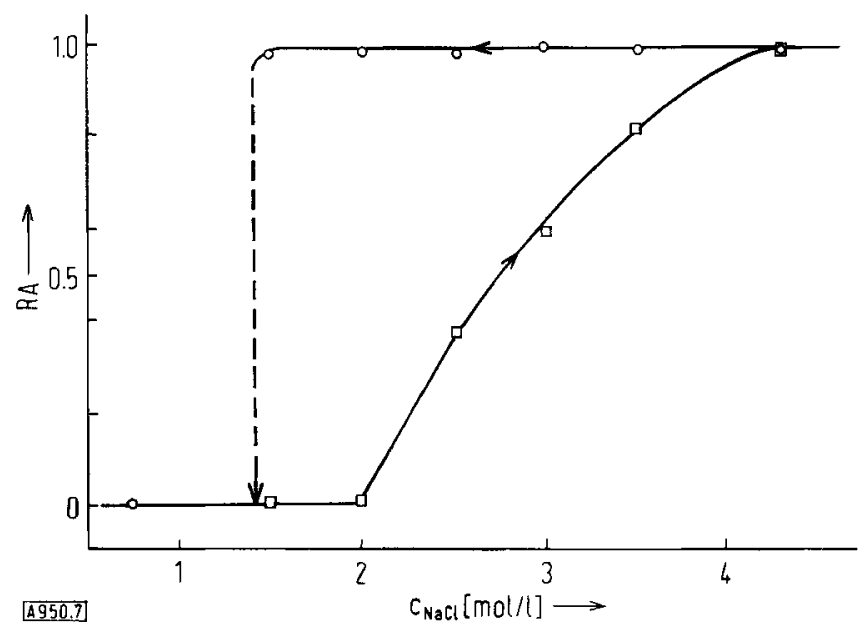

Abb. 7. Relative enzymatische Aktivitüt RA des Haloenzyms Malat-Dehydrogenase bei $30^{\circ} \mathrm{C}$; 0 : Meßwerte bei Erniedrigung, $\square$ : Melßwerte bei Erhöhung der NaCl-Konzentration $\mathrm{C}_{\mathrm{NaCl}}[43]$

Neben den bereits erwähnten Hysteresen in Nervenmembranen ${ }^{[14.15]}$ werden Metastabilitäten auch in Suspensionen von Lipidvesikeln beobachtet. Die thermischen Hysteresen solcher sphärischer Membransysteme sind an die Anwesenheit von zwei- oder dreiwertigen lonen oder auch an die Gegenwart von Acetylcholin gebunden ${ }^{[20]}$.

Hysteretische Protonenbindungskurven erhält man bei Säure-Base-Titrationen von Fragmenten der Zellhüllen von Halobakterien des Toten Meeres (vgl. Abb. 8). Die Größe dieser Membranhysterese ist von der Salzkonzentration abhängig; bei $\mathrm{NaCl}-G$ hehalten kleiner als $1 \mathrm{M}$ „schrumpft" dic Hysterese zur Gleichgewichtskurve. Wird bei konstantem $\mathrm{pH}$ die Salzkonzentration zyklisch erniedrigt und erhöht, erhält man ebenfalls einc Hystereseschleife ${ }^{[44]}$.

Wie aus Abbildung 8 ersichtlich, liegen die Nichtgleichgewichtsübergänge der Säure-Base-Hysterese bei konstanter Salzkonzentration in den $\mathrm{pH}$-Bereichen der $\mathrm{pK}$-Werte primärer Amino- und Carboxygruppen der Membranproteine (bei $3 \mathrm{M} \mathrm{NaCl}$ etwa bei $\mathrm{pH} 8.5$ und $\mathrm{pH}$ 5). Die Hysterese ist in diesen Membranfragmenten wahrscheinlich durch 


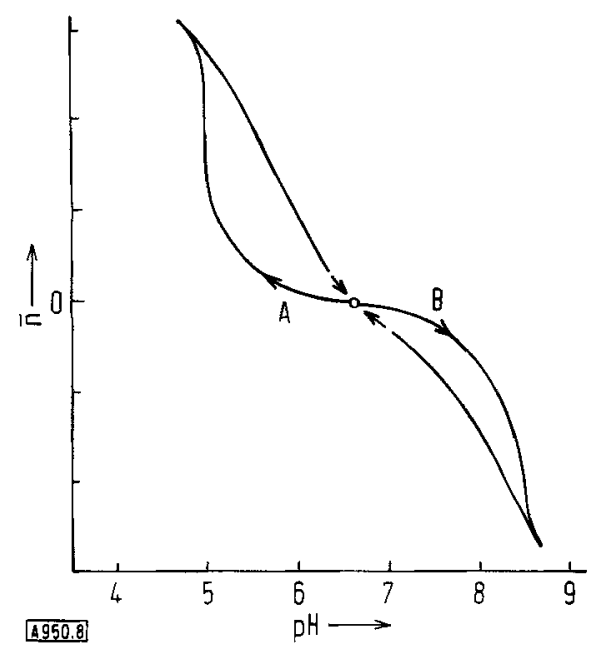

Abb. 8. Protonenbindungskurve suspendierter Membranfragmente aus den Zellhüllen von Halobakterien des Toten Meeres. $\bar{n}$, mittlere Anzahl gebundener Protonen (in willkürlichen Einheiten) als Funktion des pHWerts. A: Säure-Base-Zyklus im sauren, B: Säure-Base-Zyklus im alkalischen pH-Bereich; $20^{\circ} \mathrm{C}, 3 \mathrm{M} \mathrm{NaCl}$.

Metastabilitäten in den Ionenpaaren elektrostatisch assoziierter Seitengruppen bedingt. Elektrostatische Wechselwirkungen scheinen auch zur Ursache für die Hysterese in der Aktivität unseres Haloenzyms beizutragen. In diesem Protein beruht die Hysterese auf metastabilen Zuständen in der Assoziations-Dissoziations-Reaktion zwischen den Untereinheiten des tetrameren Enzymkomplexes ${ }^{[43]}$

Auf die kybernetische Bedeutung von Hysteresen in Membranen und katalytisch aktiven Proteinen wird in Abschnitt 8 eingegangen werden.

\section{Molekularfeld-Theorie von Hysterese}

Die allgemeine thermodynamische Beschreibung von $\mathrm{Hy}$ sterese läßt die Frage nach der physikalischen Ursache dieser Nichtgleichgewichtserscheinung offen.

Bei der molekularen Hysterese von Nucleinsäurekomplexen scheint langlebige Metastabilität durch die Kooperativität der Basenstapelung und die elektrostatischen Wechselwirkungen der Polyanionen bedingt zu sein. Tatsächlich gehen die ersten Versuche, die Hysterese im A-U-System physikalisch zu deuten, von der Annahme einer elektrostatischen Nucleationsbarriere für die Bildung protonierter (A.A)-Sequenzen aus ${ }^{[25]}$. Mit dieser Näherung ist jedoch nur eine qualitative Interpretation möglich.

Kürzlich ist eine Molekularfeld-Theorie entwickelt wor$\operatorname{den}^{[45]}$, nach der die Energiebarriere schon bei der Öffnung von (A-2U)-Segmenten in der partiell protonierten Tripelhelix auftritt. (Die zunehmende Protonierung bei der Säuretitration kompensiert einen Teil der elektrostatischen Abstoßungsenergie, die durch die Polyanionen des Tripelstranges verursacht ist, so daß eine weitere Öffnung vor Erreichen eines kritischen pH-Werts erschwert wird.)

Wir können die mittlere Freie Enthalpie $\bar{G}$ für die Stabilisierung eines Assoziats wie ein (A.2U)-Segment als eine Summe zweier Terme schreiben:

$\overline{\mathrm{G}}=-\overline{\mathrm{A}}+\overline{\mathrm{R}}$
Der Term $\bar{A}$ umfaßt alle Attraktionsbeiträge, primär kurzreichende Kräfte wie van-der-Waals- und hydrophobe Wechselwirkungen. Der zweite Term, $\bar{R}$, erfaßt ausschließlich (weitreichende) elektrostatische Abstoßungskräfte zwischen geladenen Gruppen.

Wir wollen unser „Molekularfeld“-Modell für die Tripelhelix dadurch weiter spezifizieren, daß wir die vollständig getrennten (nicht wechselwirkenden) Einzelstränge als Referenzzustand definieren, für den $\bar{R}=0$ ist. (Damit ist $\bar{R}$ die relative mittlere Freie Enthalpie eines dissoziierten Nucleotidrests in der Tripelhelix.)

In unserem Modell beruht die Energiebarriere für die Trennung assoziierter Kettenreste auf der verschiedenartigen Abhängigkeit der Größen $\bar{A}$ und $\bar{R}$ vom mittleren Abstand $\bar{d}$ zwischen gegenüberliegenden Assoziationspartnern. Wir nehmen an, daß $\bar{A}$ für $d>d_{0}$ gleich Null ist; $d_{0}$ ist der Abstand maximaler Attraktion. Im einfachen Fall von Coulomb-Repulsion ist $\bar{R}$ proportional zu $1 / \bar{d}$. Die Korrelationen zwischen $\bar{R}$ und $\bar{A}$ mit $\bar{d}$ sind in Abbildung 9 schematisch dargestellt.

Wir nehmen nun an, daß unter gegebenen experimentellen Bedingungen nur einige wenige Basenreste in der basengestapelten kooperativ stabilisierten Organisation der Tripelhelix dissoziieren können. Durch die noch geschlossenen (A.2U)-Nachbarsegmente bedingt, könnten sich solche dissoziierten Basen aber nicht weit voneinander entfernen. Ein kleiner Abstand zwischen den Assoziationspartnern bedeutet jedoch einen hohen Wert für den $\bar{R}-T e r m$. Die Dissoziation nur einiger weniger (A.2U)-Segmente würde daher einen energetisch ungünstigen Zustand schaffen; d. h. die Dissoziation trifft auf eine Nucleationsbarriere.

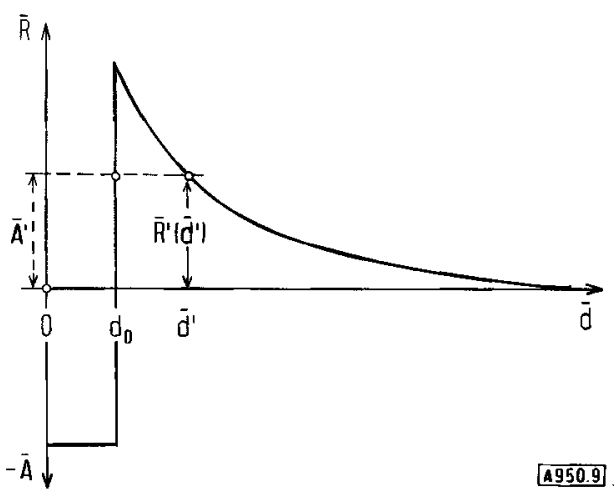

Abb. 9. Mittlere Zustandsparameter $\overline{\mathrm{R}}$ und $\overline{\mathrm{A}}$ als Funktion des Abstands $\bar{d}$ zwischen Assoziationspartnern in polyelektrolytischen Strukturen; vgl. Text.

Diese Barriere ist um so kleiner, je größer der mittlere Abstand zwischen den dissoziierten Nucleotidsegmenten werden kann. Eine solche Abstandsvergrößerung ist durch einen Anstieg in $\bar{A}$ (z. B. durch pH-Änderung) erreichbar; ein höherer Wert für $\bar{A}$ bedingt einen kleineren Wert für $\overline{\mathbf{R}}(\overline{\mathrm{d}})$. Die thermodynamische Bedingung für die Trennung der Segmente ist durch

$\bar{G}(\mathrm{~d}) \geq 0$

gegeben. Erfolgt dic Trennung bei $d=d^{\prime}$, gilt an dieser Stelle $\bar{A}^{\prime}=\bar{R}\left(d^{\prime}\right)$; siche Abbildung 9 . 
Da jedoch nun $\bar{G}\left(\bar{d}^{\prime}\right)=\bar{A}^{\prime}>0$ ist, läuft die Dissoziation und Trennung des gesamten Komplexes irreversibel $a b$. Alle Zustände für die $0<\overline{\mathrm{A}} \leq \overline{\mathrm{A}}^{\prime}$ ist, sind somit metastabil.

$\mathrm{Da} \overline{\mathrm{R}}$ von $\overline{\mathrm{d}}$ abhängt, ist $\bar{R}$ (d) offensichtlich eine Funktion des (mittleren) Bruchteils,,$\hat{f}$, geschlossener (A.2U)-Segmente einer Tripelhelix. Wenn wir annehmen, daß diese Abhängigkeit linear ist, erhalten wir mit dem Proportionalitätsfaktor $\mathrm{B}$ die Beziehung

$\overline{\mathrm{R}}=\mathrm{B} \cdot \overline{\mathrm{f}}$

Aus Gl. (31) ist leicht ersichtlich, daß der Maximalwert für $\bar{R}$ bei $\overline{\mathrm{f}}=1$ ist, während $R=0$ für $\overline{\mathrm{f}}=0$ gilt.

Da jedes Segment der Tripelhelix den weitreichenden elektrostatischen Kräften (oder dem molekularen Feld) aller übrigen Kettensegmente unterliegt, können wir Gl. (31) die Molekularfeld-Näherung für unser Modell nennen.

In dem Bereich experimenteller Bedingungen, in dem $\overline{\mathrm{G}}$ von $\bar{d}$ und damit von $\mathrm{f}$ abhängt, können wir den Gesamtwert $G_{t o t}$ der Freien Enthalpie als Funktion von $f$ nach folgender Beziehung berechnen:

$G_{1 m}=-\bar{A} \cdot f+(1-f) \cdot B \cdot f+R T[f \ln f+(1-f) \ln (1-f)]$

Es kann nun gezeigt werden, daß die Zustandsfunktion $\mathrm{G}_{\text {to }}$ für $\mathrm{B}>2 \mathrm{RT}$ zwei Minimumwerte hat; einer ist dem metastabilen Zustand, der andere dem thermodynamischen Gleichgewichtszustand zugeordnet. Ein solches Energiediagramm mit zwei Minima ist schon von Borelius zur Deutung von Hysterese benutzt worden ${ }^{[22]}$.

Ein wichtiges Ergebnis der Theorie ist eine Bedingung für das Auftreten von thermodynamisch metastabilen $\mathrm{Zu}$ ständen:

B $>2$ RT

Für das (A.2U)-Modellsystem gilt die in Gl. (32) ausgedrückte Ungleichung für niedrige Temperaturen $\left(<50^{\circ} \mathrm{C}\right)$ während für die (A.U)-Doppelhelix $\mathrm{B}<2 \mathrm{RT}$ ist. Bei der Curie-Temperatur $T_{c}$ gilt $B=2 R T_{c} ; d$. h., für $T \geq T_{c}$ verschwindet die Hysterese und man erhält nur die Gleichgewichts!urve ${ }^{[8]}$.

Die Hystereseschleifen, die bei Membranen und Proteinkomplexen hoher Oberflächenladungsdichte beobachtet werden, können in ähnlicher Weise wic das Polynucleotidsystem mit einer Molekularfeld-Theorie molekular interpretiert werden.

\section{Hysterese als analytische Methode}

Die extreme Langlebigkeit der Metastabilität einiger Biopolymer- und Membranstrukturen gestattet es, Hysterese als analytische Methode zur Untersuchung der Verteilung von Strukturuntereinheiten zu benutzen. Wie schon erwähnt, deutet das Auftreten von Scanning-Kurven Domänstrukturen an. Durch Analyse dieser Scanning-Kurven kann im Prinzip die Verteilungsfunktion der Domänen eines Systems und ihrer charakteristischen Übergangspunkte ermittelt werden. Ist diese Funktion bekannt, ist das Scanningverhalten voraussagbar ${ }^{[46.47]}$.

Eine solche Analyse basiert auf Domäntheorien, denen das Preisach-Modell zugrundeliegt ${ }^{[48]}$. Im einfachen Fall voneinander unabhängiger Domänen läßt sich nach Ever$e t t^{[46]}$ die Domän-Verteilungsfunktion $\phi(x)$ relativ einfach aus einer begrenzten Zahl von Scanning-Kurven bestimmen, wenn das mittlere Ausmaß $\xi_{i}$ der irreversiblen Zustandsänderungen (d.h. der Bruchteil umgewandelter Domänen)als Funktion des äußeren Parameters x ermittelt werden kann. Wird jeder Domäne ein unterer und oberer Umwandlungspunkt $x_{1}$ und $x_{u}$ (entsprechend $x$ (II) und $x(I)$ in Abb. 2) zugeordnet, ist $\phi(x)$ nach der Beziehung

$\phi(x)=-\frac{\partial^{2} \xi}{\partial x_{0} \partial x_{1}}$

aus experimentellen $\xi_{\mathrm{i}}(\mathrm{x})$-Werten bestimmbar ${ }^{[46]}$.

Die Zuordnung von unteren und oberen $x$-Grenzen für die Scanning-Schritte gestattet es, einen Scanningprozeß in einfacher Weise zu beschreiben. Danach werden die Wege, auf dem ein Punkt der Hysterese erreicht wurde, in einer Domän-Komplexion zusammengefa $\left[\mathrm{t}^{[+6]}\right.$. Diese Funktion beschreibt die wegabhängige Vorgeschichte in analoger Weise wie die Nachwirkungsfunktionen, die Boltzmann 1876 zur Beschreibung elastischer Nachwirkungen in Abhängigkeit von der Deformationsvorgeschichte eingeführt hat ${ }^{[49]}$. Somit ist die Everettsche Domän-Komplexion eine Art von Gedächtnisfunktion, und die DomänVerteilungsfunktion beschreibt die "Gedächtniskapazität" eines Multidomänsystems.

Um zu prüien, ob ein System unabhängiger Domänen vorliegt, dient ein Schlüsseltheorem der Everett-Theorie: die Flächen von $\xi_{\mathrm{i}}(\mathrm{x})$-Scanningschleifen zwischen denselben $x$-Grenzen müssen kongruent sein. Sind dic $\xi_{i}$-Wertc jedoch nicht ermittclbar, kann cine Modifizierung dieses Theorems durch Enderby ${ }^{1+71}$ herangezogen werden.

Danach werden die gemessenen Scanningschleifen (z. B. $\bar{\alpha}(\mathrm{pH})$-Kurven) zwischen gleichen $\mathrm{x}_{\mathrm{u}}$ - und $\mathrm{x}_{1}$-Werten vergli-

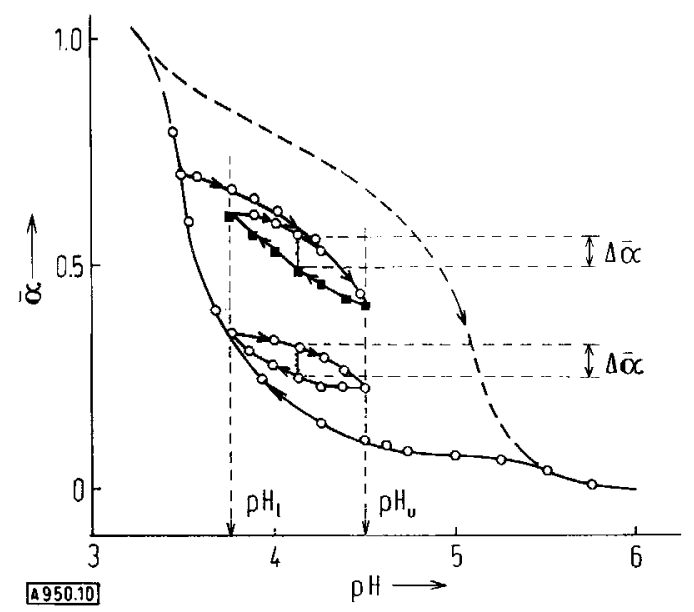

Abb. 10. Scanningschleifen in einem (A-2U)-System breiter Molekulargewichtsvertcilung. $\mathrm{pH}_{1}$ : untere, $\mathrm{pH}_{\mathrm{u}}$ : obere pll-Grcize der Schleifen. Vergleich der $\Delta \bar{\alpha}$-Werte der Schleifen bei gleichem pH-Wert: vgl. Abb. 4 und Texi. 
chen. In einem System, in dem der Zustand einer Domäne von dem der übrigen unabhängig ist, müssen dic Ordinatenabschnitte bei gleichem $x$-Wert innerhalb der Schleifen gleich scin.

Wenden wir dieses Theorem auf Scanningschleifen im (A.2U)-System mit relativ breiter Molekulargewichtsverteilung an, so erhalten wir das in Abbildung 10 gezeigte Bild. Es ist ersichtlich, daß die Schleifenabschnitte $\Delta \bar{\alpha}(\mathrm{pH})$ innerhalb von $10 \%$ gleich sind. Danach scheinen die (A.2U)-Komplexe sich unabhängig zu verhalten.

Nehmen wir an, daß (A-2 U)-Komplexe verschiedener Molekulargewichte verschiedene Stabilität besitzen, dann ergibt die Differentiation der Hysteresegrenzkurve die relative Verteilung dieser Stabilitäten und die relative Anzahl der Tripelhelices verschiedenen Molekulargewichts entlang der pH-Skala. Damit ist die Analyse des Scanningverhaltens im Prinzip eine Methode, die Verteilung von Molekulargewichten in Biopolymergemischen zu bestimmen.

\section{Durch elektrische Impulse bewirkte Konformationsänderungen in polyelektrolytischen Systemen}

Säure-Base-Titrationen sind eine Standardmethode, die Thermodynamik von Strukturänderungen in ionisierbaren Systemen zu untersuchen. Werden die Titrationen bei verschiedenen Ionenstärken ausgeführt, ist es möglich, die elektrostatischen Beiträge in der Energetik der Strukturumwandlungen zu bestimmen. Solche elektrostatischen Beiträge sind bei polyelektrolytischen Systemen besonders groß.

Nach Gl. (19) und (20) gilt für homogene polyelektrolytische Systeme

$\log \frac{1-\alpha}{\alpha}=p H-\mathrm{pK}^{0}-\frac{\mathrm{z} \cdot \mathrm{e} \cdot \psi}{2.3 \mathrm{kT}}$

In dieser Beziehung ist ersichtlich, daß $\alpha$ sowohl durch pH-Änderung als auch - bei konstantem $\mathrm{pH}$ - durch eine Änderung des elektrischen Potentials $\psi$ beeinflußbar ist. Im allgemeinen ist $\psi$ durch das intramolekulare elektrische Feld bestimmt. Dieses innere Feld ist durch den Ionisationsgrad (Protonierungsgrad) und durch die Ionenstärke des Mediums aber auch durch externe elektrische Felder beeinflußbar, denen das System ausgesetzt sein kann.

In unserem Hysterese-Modellsystem kann das (A - 2 U)-Protonierungs-Vorgleichgewicht im $\mathrm{pH}$-Bereich vor dem irreversiblen Phasenübergang bei $\mathrm{pH}_{\mathrm{m}}^{\prime}$ durch

$\log \frac{1-\alpha_{3}}{\alpha_{3}}=p I I-\mathrm{pK}_{\mathrm{AMP}}^{0}+\frac{\mathrm{e} \cdot \psi}{2.3 \mathrm{kT}}+\frac{\Delta_{\mathrm{d}} \mathrm{G}_{3}}{2.3 \mathrm{kT}}$

beschrieben werden; $\mathrm{pK}_{\mathrm{AMP}}^{0}$ ist der pK-Wert des Adenosinmonophosphats, und $\Delta_{d} G_{3}$ entspricht der pK-Verschiebung durch den Bruchteil der (A)-Segmente, die während der Protonierung aus dem (A.2U)-Verband dissoziieren; vgl. Gl. (28). ( $\psi$ ist primär durch die negativ geladenen
Phosphate bestimmt und damit negativ!) Bei $\alpha=\alpha_{3}^{\prime}$, entsprechend $\mathrm{pH}=\mathrm{pH}_{\mathrm{m}}^{\prime}$, erfolgt die irreversible Assoziation zu (A.A)-Sequenzen.

Eine Änderung des $\psi$-Terms, die zu $\alpha>\alpha_{3}^{\prime}$ führt, kann z. B. bei konstantem $\mathrm{pH}$ durch eine Änderung der Ionenstärke bewirkt werden. Tatsächlich läßt sich die irreversible Bildung der (A.A)-Doppelhelix aus der metastabilen (A.2U)-Tripelhelix durch Verkleincrung der Salzkonzentration auslösen ${ }^{|3.3|}$. Kürzlich haben wir gefunden, daß elektrische Impulse (der anfänglichen Feldintensität von etwa $20 \mathrm{kV} / \mathrm{cm}$, dic exponentiell mit einer Relaxationszeit von etwa $10 \mu \mathrm{s}$ abfällt) diesen Konformationsuibergang direkt induzieren können ${ }^{[50]}$.

Für die elektrische Auslösung solcher Konformationsänderungen wurde ein Polarisationsmechanismus vorgeschlagen, nach dem das äußere elektrische Feld die Ionensphäre des polyelektrolytischen (A.2U)-Komplexes verschiebt und dadurch ein Dipolmoment erzeugt. Am negativen Ende dieses Makrodipols ist die Ionenwolkenabschirmung der negativen Phosphatgruppen reduziert. Das bewirkt Abstoßung zwischen den Enden der Polyanionen und führt schließlich zum Aufwinden der Tripelhelix ${ }^{[50]}$.

Ein ähnlicher Mechanismus ist für die Auslösung von Permeabilitätsänderungen in vesikulären Membranen hoher Oberflächenladung durch elektrische Impulse anzunehmen. Danach erhöht die Verschiebung der abschirmenden Ionensphäre den mittleren Abstand zwischen gleichsinnig ionisierten Gruppen auf der Membranoberfläche. Das wiederum erhöht die Durchlässigkeit der Membran. In dieser Weise ist die durch elektrische Impulse ausgelöste Freisetzung von biogenen Aminen aus suspendierten ChromaffinGranula gedeutet worden ${ }^{[51]}$.

Die elektrischen Impulse, die bei Lösungen des (A.2U)Komplexes und bei Vesikelsuspensionen verwendet wurden, sind mit den Spannungsstößen der Aktionspotentiale in Nervenmembranen vergleichbar. Die elektrischen Felder, die über die Nervenmembran auftreten können, ändern sich bei der Erregung in Richtung und Intensität von ca. $-70 \mathrm{kV} / \mathrm{cm}$ auf ca. $+50 \mathrm{kV} / \mathrm{cm}$. Wir können annehmen, daß nicht nur in der Membran, sondern auch in ihrer unmittelbaren Nähe und (vorübergehend) auch über den synaptischen Zwischenraum elektrische Felder existieren. Daher könnte ein biopolyelektrolytisches System, das solchen Feldern ausgesetzt ist, in ähnlicher Weise elektrisch beeinflußt werden wie der (A.2U)-Komplex oder die Chromaffin-Vesikeln.

Elektrisch induzierte Konformationsänderungen in Makromolekülen und Membranen werden als möglicher Mechanismus für elektrisch kontrollierte Regelvorgänge und insbesondere für die Aufzeichnung elcktrischer Signale diskuticrt ${ }^{11.50]}$. Vor diesem Hintergrund betrachten wir gerichteteStrukturübergänge, die in metastabilen polyclcktrolytischen Systemen durch elektrische Impulse ausgelöst werden, als Modellreaktionen für den Aufdruck von Nervenimpulsen in die Strukturen, die bei einer physikalischen Aufzeichnung von Information beteiligt sind.

Von physiologisch-chemischen Studien ist bekannt, daß Nervenstimulierung den Metabolismus innervierter Zellen beschleunigt; so steigt z. B. die RNA- und Proteinsynthese 
bei elektrischer Aktivität der Nervenzelle an. Der Mechanismus der Kopplung zwischen elektrischer Aktivität und chemischer Reaktion ist noch unbekannt. Angesichts der Hysteresekapazität von rRNA, die ähnlich wie beim A-U-System elektrisch beeinflußt werden könnte, liegt die Frage nahe, ob ribosomale Aktivität über rR NAMetastabilitäten durch elektrische Aktivität der Zelle „regelbar" ist.

Auch andere Zellvorgänge unterliegen dem Einfluß der Nervenaktivität. So wird die Sekretion von Hormonen und Neuroeffektoren (Transmittersubstanzen) bei Nervenstimulierung erheblich verstärkt. Die Depolarisierungsspannung, die bei der Chromaffin-Zelle zu verstärkter Hormonausschüttung führt, ist mit der Anfangsintensität der Spannungsstöße vergleichbar, die bei suspendierten Chromaffin-Granula den Ausfluß von Neuroaminen verursachen. Daher liegt es nahe, einen Mechanismus anzunehmen, bei dem die Nervenaktivität direkt Neurosekretionen über Permeabilitätsänderungen in den Membranen der Speichervesikeln in Intensität und Ausmaß steuern. Solche impulsinduzierten Durchlässigkeitsänderungen von Mcmbranen, kombiniert mit der Freisetzung von Neuroeffektoren, sind wahrscheinlich Elementarprozesse bei dem Vorgang der synaptischen Bahnung in der funktionellen Entwicklung des Zentralnervensystems.

Bemerkenswert ist die Beobachtung eines Schwellenwerts der Impulsintensität von etwa $18 \mathrm{kV} / \mathrm{cm}$ sowohl für die Auslösung der Konformationsübergänge im (A.2U)-Modellsystem wie auch für die Permeabilitätsänderung der Vesikelmembran. Der Schwellenwert der Depolarisierungsspannung, der in Nervenmembranen erreicht werden muß, um Aktionspotentiale auszulösen, ist etwa $20 \mathrm{mV}$; auf cine durchschnittliche Membrandicke von $100 \AA$ bezogen, entsprechen $20 \mathrm{mV}$ einer Feldstärke von $20 \mathrm{kV} / \mathrm{cm}$. Diese Ähnlichkeit der Schwellenwertspannungen hat die Diskussion um eine chemische Kontrolle der Ionenfluisse des Aktionspotentials neu belebt. Danach werden impulsinduzierte Konformationsänderungen von Membrankomponenten als Mechanismus für die Aktivierung von Acetylcholin vorgeschlagen ${ }^{152]}$. Die relativ hohen Beträge in der Änderung der Wärmeenergie, die ein Aktionspotential begleiten ${ }^{[53]}$, deuten eine Beteiligung von chemischen Reaktionen während der Nervenaktivität an. Es sei daran erinnert, dál chemische Reaktionen und Konformationsübergänge, die irreversibel erfolgen, von Wärmeentwicklung begleitet sind, die aus der Dissipation Freier Enthalpie herrührt. In diesem Zusammenhang ist es bemerkenswert, daß in einem Depolarisations-Polarisations-Zyklus der Nervenmembran während des Aktionspotentials insgesamt Wärme erzeugt wird ${ }^{1531}$. Es ist eine offene Frage, ob diese freigesetzte Wärmeenergie durch Membrankomponenten verursacht wird, die ihre Konformation entlang einer Hystereseschleife ändern. Vgl. dazu die von Tasaki beobachtete Hysterese in der Membran des Tintenfisch-Riesenaxons ${ }^{[14]}$.

\section{Hysterese als Basis chemischer Oszillationen}

Die zirkular vektorielle Eigenschaft der Hysterese ist eine potenticllc Basis für chemische Oszillationen. Mikroskopi- sche chemische Oszillationen sind als Ursache makroskopischer biologischer Uhren von fundamentaler Wichtigkeit $^{[10.54]}$.

Das Prinzip molekularer, auf Hysterese beruhender Periodizität ist die Kopplung eines chemischen Reaktionsflusses mit einem Diffusionsprozeß. Voraussetzung für das Entstehen von Oszillationen ist eine Hysterese entweder im Reaktionsfluß oder im Diffusionsfluß. Zum Beispiel wird eine periodische Konzentrationsänderung eines Reaktionsprodukts durch einen Reaktanden verursacht, der sowohl an einer "hysteretisch“ katalysierten Reaktion als auch an einem Diffusionsprozeß (z. B. durch eine Membran kontrolliert) teilnimmt. (Theoretisch kann auch eine Kopplung zwischen Autokatalyse und Enzymreaktion zu Oszillationen führen; vgl. z. B. ${ }^{[55]}$.)

Periodische Änderungen, die auf Chemodiffusion beruhen, sind seit langem bei der Heterokatalyse von Gasreaktionen an Metalloberflächen bekannt; vgl. z. B. ${ }^{[56]}$. Jedoch ist erst kürzlich wahrscheinlich geworden, daß auch auf der Ebene einzelner Katalysatormoleküle in homogener Lösung solche periodischen Vorgänge stattfinden können. In der halophilen Malat-Dehydrogenase begegnen wir einem Beispiel eines solchen hysteretischen Katalysators ${ }^{\mid+3]}$.

Periodische Änderungen von Diffusionsflüssen können durch Membranen bewirkt werden, die ihre Permeabilitäl hysteretisch ändern. Die Zellhüllen der Halobakterien des Toten Meeres könnten ihre Durchlässigkeit als Funktion der Salzkonzentration in dieser Weise variieren, wenn die Strukturänderungen, die durch die Protonenbindungshysterese angezeigt sind, Permeabilitätsänderungen der Zellmembran verursachen. Aus experimentellen Ergebnissen von Ginzburg et al. ${ }^{[57]}$ kann geschlossen werden, daß diese Bakterien ihren Salzgehalt periodisch ändern. Es ist Gegenstand weiterer Untersuchungen, ob eine Korrelation zwischen Membranhysterese und hysteretischer Aktivitätsänderung der Enzyme besteht, die die „Salzuhr“ dieser Organismen bedingen könnte.

Das Prinzip hysteretischer Chemodiffusion sei am Modell eines Enzyms erläutert, welches durch Substratbindung bei einer kritischen Substratkonzentration $S_{2}$ katalytisch aktiv wird. Das Substrat soll mil konstanter Geschwindigkeit durch den Reaktionsraum fließen; d. h. der Diffusionsfluß $J_{d}$ des Substrats ist konstant.
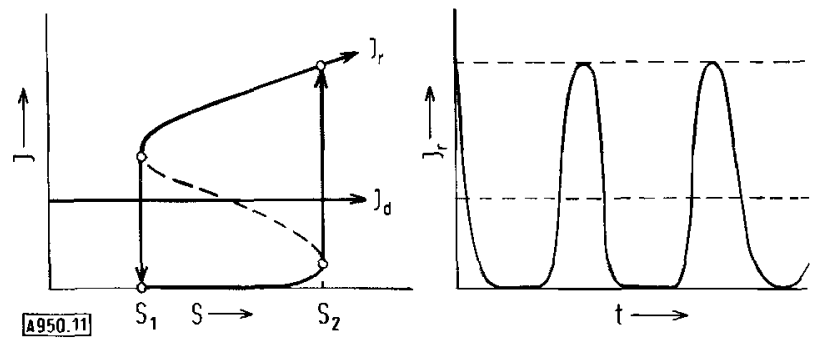

Abb. 11. Kopplungsschema zur Umwandlung von Stationaritat in Periodizität durch hysteretische (hemodiffusion. S: Substratkonzentration: $J_{d}$ : Diffusionsflu $3 ; I_{r}$ : Reaktionsfluß.

In der schematischen Darstellung von Abbildung 11 ist ersichllich, daß für $S>S_{2}$ der Reaktionsfluß $J_{r}(S)$ größer 
als der Diffusionsfluß $\mathrm{J}_{\mathrm{d}}(\mathrm{S})$ ist. Der Substratabbau ist damit schneller als der Zufluß. Dadurch verarmt der Reaktionsraum an Substrat, bis bei einer unteren kritischen Substratkonzentration $\mathrm{S}_{1}$ das Enzym inaktiv wird; jetzt ist der Reaktionsfluß kleiner als der Diffusionsfluß, und das Substrat kann sich wieder ansammeln bis $\mathrm{S}>\mathrm{S}_{2}$ wird. Bei $S_{2}$ beginnt der Zyklus von neuem. Das Ergebnis ist eine periodische Änderung des Reaktionsflusses und damit der Produktkonzentration ${ }^{[10]}$.

In ähnlicher Weise kann eine hysteretische Permeabilitätsänderung einer Membran cinen stationären Fluß in einen periodischen Fluß umwandeln ${ }^{[12]}$. In dieser Umwandlerfunktion liegt nun dic fundamentale Bedeutung hysteretischer Makromoleküle und Membranen.

Während die klassische Biologie lebende Organismen durch stationäre Zustände (Homöostasie) charakterisiert, versuchen moderne Theorien dem tatsächlichen dynamischen Verhalten biologischer Organisationen durch eine Beschreibungsweise näher zu kommen, die Quasi-Stationarität, d.h. Oszillationen um einen stationären Mittelwert, beinhaltet ${ }^{[58]}$. Hysterese stellt nun einen einfachen, energetisch günstigen Mechanismus dar, auf molekularer Ebene Stationarität in Periodizität umzuwandeln.

\section{Schlußbemerkung}

Die Entdeckung von Konformations-Metastabilität in Nucleinsäuren, Proteinen und Membranen hat $\mathrm{zu}$ einem neuen kybernetischen Zugang der dynamischen Deutung zellulären Geschehens geführt. Es sei angemerkt, daß die Dynamik biologischer Organisation und Funktion wahrscheinlich durch eine allgemeine Netzwerktheorie beschreibbar ist, die kürzlich von Oster, Perelson und Katchalsky entwickelt wurde ${ }^{[59]}$. In dieser Näherung, Netzwerkthermodynamik genannt, sind „Transducer" als Kopplungseinheiten zwischen den Netzwerkelementen eingeführt worden. Es scheint nun, daß Makromoleküle und Membranen, die metastabile Strukturen ausbilden können, als elementare kybernetische Einheiten für allgemeine Umwandlungs- (oder Transducer-)Prozesse und insbesondere für Regulations- und Gedächtnisvorgänge besonders geeignet sind. Metastabilen polyelektrolytischen Systemen kommt in der Fähigkeit, „Information“, die durch Ionenströme oder elektrische Impulse aufgeprägt wurde, molekular zu speichern, besondere Bedeutung für eine schnelle und energiearme Informationsübermittlung zu.

In einem dynamischen Bild zellulärer Organisation und Funktion scheint wenig Raum für perfekte Stabilität zu sein, wie sie ideale Gleichgewichtszustände charakterisiert. Selbst einfache Stationarität erscheint nicht ausreichend für eine allgemeine Beschreibung zentraler biologischer Vorgänge, wie z.B. die Regulation und Kontrolle von Enzymkatalyse, Membrantransport, Informationsuibermittlung oder Selbstorganisation ${ }^{160.611}$. Nichtgleichgewichtsnäherungen, die metastabile Zustände und molekulare Hysterese einbcziehen, - so glaube ich - können zu einem besseren Verständnis der komplexen Dynamik fundamentaler Lebenserscheinungen beitragen.
Der Stiftung Volkswagenwerk danke ich für ein Forschungsstipendium am Weizmann Institute of Science in Israel.

Eingegangen am 5. März 1973

[A 950]

[1] A. Katchaisky, Endeavour 12 (4), 90 (1953).

[2] A. Karchalsky, Biophys. J. 4, 9 (1964).

[3] J. Stauff: Kolloidchemie. Springer-Verlag, Berlin 1960.

[4] D. H. Everett in E. A. Flood: The Solid Gas Interface, Vol. 2. Marcel Dekker, New York 1967, S. 1055.

[5] A. Ewing, Proc. Roy. Soc. London 33, 21 (1881); Phil. Trans. Roy. Soc. London 523 (1885).

[6] R. A. Cox, A. S. Jones, G. E. Marsh u. A. R. Peacocke, Biochim. Biophys. Acla 21, 567 (1956).

[7] A. Revzin, E. Neumann u. A. Katchalsky, J. Mol. Biol., im Druck. [8] E. Neumann u. A. Kalchalsky, Ber. Bunsenges. Phys. Chem. 74, $868(1970)$

[9] A. Katchaisky in S. Devons: Biology and the Physical Sciences. Columbia University Press, New York 1969, S. 267.

[10] A. Katchalsk! u. R. Spangler, Quart. Rev. Biophys. 1, 127 (1968).

[11] A. Katchalsky u. E. Neumann, Int. J. Neurosci. 3, 175 (1972).

[12] A. Katchalsk! u. G. Oster in D. C. Testeson: The Molecular Basis of Membrane Function. Prentice-Hall, Englewood Cliffs, N. I. 1969, S. 1

[13] A. Sollberger: Biological Rhythm Research. Elsevier, Amsterdam 1965.

[14] 1. Tasaki, W. Barry u. L. Carnay in F. Snell et al.: Physical Principles of Biological Membranes. Gordon-Breach, New York 1970, S. 17.

[15] H. R. Clark u. A. Strickholm. Nature 234, 470 (1971).

[16] G. Adam in F. Snell a al.: Physical Principles of Biological Membranes. Gordon-Breach, New York 1970, S. 35.

[17] Vgl. z. B.: S. Lifson u. B. H. Zimm, Biopolymers 1, 15 (1963).

[18] E. Neumann u. T. Ackermann, J. Phys. Chem. 73, 2170 (1969).

[19] F. M. Pohl, Angew. Chem. 84, 931 (1972); Angew. Chem. internat. Edit. 11, 894 (1972).

[20] H. Träuble, Naturwissenschaften 58, 277 (1971).

[21] T. L. Hill, J. Chem. Phys. 17, 520 (1949).

[22] G. Borelius, Ann. Phys. (Leipzig) 20, 57 (1934); Proc. Phys. Soc. London 49, 77 (1937).

[23] A. Katchalsky u. P. F. Curran: Nonequilibrium Thermodynamics in Biophysics. Harvard University Press, Cambridge, Mass. 1965.

[24] l. Prigogine: Étude Thermodynamique des Processus Irreversibles. Desour, Liège 1947.

[25] A. Katchalsky, A. Oplatka u. A. Litan in T. Hayashi u. A. G. Szent G!örg!'i: Molecular Architecture in Cell Physiology. Prentice-Hall, Englewood Cliffs, N. J. 1966, S. 3; A. Katchalsky u. A. Oplatka, Neurosci Res. Symp. Sum. 1, 352 (1966).

[26] Z. Alexandrowiczs u. A. Katchalsky, J. Polymer Sci. A1, 3231 (1963).

[27] G. Felsenfeld u. A. Rich, Biochim. Biophys. Acta 26, 457 (1957). [28] R. C. Warner u. E. Brestow, Proc. 4th Int. Congr. Biochem. Vienna 9,157 (1958).

[29] R. F. Steiner u. R. F. Beers, Jr., Biochim. Biophys. Acta 33, 470 (1959).

[30] R. A. Cox, Biochim. Biophys. Acta 68, 401 (1963).

[31] V. Vetterl u. W. Guschlbauer, Arch. Biochem. Biophys. 148, 130 (1972).

[32] H. J. Hinz, O. J. Schmitz u. T. Ackermam, Biopolymers 7, 611 (1969).

[33] M. Spodheim u. E. Neumann, Progr. Rep. Weizmann Inst. Israe (1972).

[34] W. Guschlbauer, D. Thiele, M.-T. Sarocchi u. C. Marck, Symp. Orléans 1972 .

[35] D. Thicle u. W. Guschlbater, Biopolymers 8,361 (1969).

[36] D. Barszcz u. D. Shugar, Eur. J. Biochem. 5.91 (1968)

[37] E. M. Gotschalk, E. Kopp u. A. G. Lezius, Eur. J. Biochem. 24 168 (1971)

[38] J. Pitha, P. M. Pitha u. P. O. P. Tso. Biochim. Biophys. Acla $204,39(1970)$.

[39] Vg], z. B.: F. Travers, A. M. Michelson u. P. Douzou, Biochim. Biophys. Acta 199. 29 (1970). 
[40] R. A. Cox u. A. Katchalsky, Biochem. J. 126, 1039 (1972).

[41] T. M. Schuster, B. R. Jennings u. G. Spach in: Nobel Symp. II. Almquist U. Wiksell, Stockholm 1969.

[42] Vgl. z. B.: S. N. Timasheff in A. Veis: Biological Polyelectrolytes. Marcel Dekker, New York 1970.

[43] M. Mevarech u. E. Neumann, Progr. Rep. Weizmann Inst. Israel (1972).

[44] E. Neumarn, noch unveröffentlicht.

[45] G. Weisbuch u. E. Nelumann, Biopolymers, im Druck.

[46] D. H. Evereh, Trans. Faraday Soc. 51,1551 (1955).

[47] J. A. Enderb!, Trans. Faraday Soc. 51, 835 (1955).

[48] Vgl. 2. B.: E. Kneller: Ferromagnetismus. Springer-Verlag, Berlin 1962.

[49] L. Bolrzmann, Poggendorfs Ann. Erg. 7, 624 (1876).

[50] E. Neumann u. A. Katchalsky, Proc. Nat. Acad. Sci. U. S. 69 , 993 (1972).

[51] E. Neumann u. K. Rosenheck, J. Membrane Biol. 10, 279 (1972).
[52] E. Neumann, D. Nachmansohn u. A. Katchalsky. Proc. Nat. Acad. Sci. U. S. 70,727 (1973).

[53] B. C. Abbot, A. V. Hill u. J. V. Howarth, Proc. Roy. Soc. London B 148, 149 (1958); J. V. Howarh, R. D. Keynes u. J. M. Ritchie, J. Physiol. (London) 194.745 (1968).

[54] A. Katchalsky, Neurosci. Res. Program Bull. 9, 397 (1971).

[55] B. Edelstein, J. Theor. Biol. 29, 57 (1970).

[56] H. Beusch, P. Figuth u. E. Wicke. Chem.-Ing.-Tech. 44, 445 (1972).

[57] M. Ginzburg, L. Sachs u. B. Z. Ginzburg, J. Gen. Physiol. 55, 187 (1970).

[58] A. Karchalsky in G. C. Quarton, T. Melnechut u. F. O. Schmitt: The Neurosclences, Vol. 1. The Rockefeller Univ. Press, New York 1967, S. 326.

[59] G. Oster, A. Perelson u. A. Katchalsky, Nature 234, 393 (1971); Quart. Rev. Biophys. im Druck.

[60] M. Eigen, Naturwissenschaften 58, 465 (1971).

[61] I. Prigogine u. G. Nicolis, Quart. Rev. Biophys. 4. 107 (1971).

\section{ZUSCHRIFTEN}

\section{Alkyl- und Aryl-hydridobis(o-phenylendioxo)- phosphat(1-), ein stabiles Spiroanion mit hexakoordiniertem Phosphor $\left.{ }^{[* *}\right]$}

Von Markus Wieber und Keywan Foroughi ${ }^{* 1}$

Reaktionen von Derivaten des fünfbindigen Phosphors verlaufen oft über Zwischenstufen mit hexakoordiniertem P-Atom ${ }^{11}$. Uns gelang es erstmals, bei der Überführung vondreibindigem in fünfbindigen Phosphor durch die Reaktion $^{12]}$

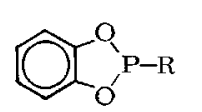

(1)<smiles>Oc1ccccc1O</smiles>
$\mathrm{OH}$

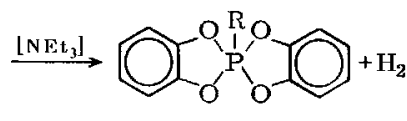

(2)

$$
\mathrm{R}=\mathrm{CH}_{3}, \mathrm{C}_{6} \mathrm{H}_{5}
$$

ein stabiles Zwischenprodukt mit hexakoordiniertem Phosphor zu isolieren.

Methyl(oder phenyl)hydridobis(o-phenylendioxo)phosphat $(1-)$ kann präparativ durch Umsetzung der 1,3,2-Benzodioxaphosphole (1) mit Brenzcatechin und Triäthylamin im Molverhältnis $1: 1: 1$ als Triäthylammonium-Salz (3) erhalten werden.

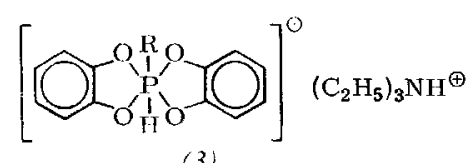

(3)

$$
\mathrm{R}=\mathrm{CH}_{3}, \mathrm{C}_{6} \mathrm{H}_{5}
$$

Wie aus den NMR-Spektren (Tabelle 1) hervorgeht, liegen die Produkte auch im polaren Lösungsmittel in der Spiroform mit der Koordinationszahl 6 für das P-Atom

[*] Prof. Dr. M. Wieber und cand. chem. K. Foroughi Institut für Anorganische Chemie der Universität 87 Würzburg. I andwehr

[**] Wir danken Herrn Dr. W. Buchmer für die Diskussion der NMR Spektren, der Deutsehen Forschungsgemeinschaft und dem Fonds der Chemischen Industrie für die Unterstützung dieser Arbeit. und einer $\mathrm{P}-\mathrm{H}-\mathrm{Bindung}{ }^{\mathrm{3} \mid}$ vor und nicht in einer eventuell diskutierbaren $^{[4,5]}$ offenen Form mit der Koordinationszahl 5 und einer $\mathrm{O}-\mathrm{H}$-Bindung.

Reines (3) zersetzt sich exotherm ${ }^{161}$ bei $83^{\circ} \mathrm{C}\left(\mathrm{R}=\mathrm{CH}_{3}\right)$ bzw. $128^{\circ} \mathrm{C}\left(\mathrm{R}=\mathrm{C}_{6} \mathrm{H}_{5}\right)$ unter Dehydrierung, d.h. Freisetzen stöchiometrischer Mengen Triäthylamin sowie Wasserstoff und Bildung des Spirophosphorans (2).

$$
\text { (3) } \rightarrow(2)+\mathrm{TI}_{2}+\left(\mathrm{C}_{2} \mathrm{H}_{5}\right)_{3} \mathrm{~N}
$$

Diese Zersetzungsreaktion ist die Ursache der Katalysator-

\begin{tabular}{|c|c|c|c|}
\hline \multicolumn{4}{|c|}{${ }^{1} \mathrm{H}-\mathrm{NMR}(100 \mathrm{MHz}$, TMS extern, $\delta$ in ppm. $\mathrm{J}$ in $\mathrm{Hz})$} \\
\hline $\mathrm{R}=\mathrm{CH}_{3}:$ & $\begin{aligned} \delta & =0.6 \\
& =6.4 \\
& =6.0\end{aligned}$ & $\begin{array}{r}3 \mathrm{H} / \mathrm{d} \\
/ \mathrm{d} \\
1 \mathrm{H} / \mathrm{d} \\
/ \mathrm{q} \\
8 \mathrm{H} / \mathrm{m}\end{array}$ & $\begin{array}{l}J_{P-X-H 3}=107 \\
J_{H-P-C-H I_{3}-}=1.5 \\
J_{P_{-H}}=620 \\
J_{H_{3}-C-P-H}=1.5\end{array}$ \\
\hline $\mathrm{R}=\mathrm{C}_{6} \mathrm{H}_{5}:$ & $\begin{aligned} \delta & =6.7 \\
& =6.9 \\
& =6.0\end{aligned}$ & $\begin{array}{l}1 \mathrm{H} / \mathrm{d} \\
5 \mathrm{H} / \mathrm{m} \\
8 \mathrm{H} / \mathrm{m}\end{array}$ & $J_{P-H}=642$ \\
\hline
\end{tabular}
wirkung von Triäthylamin im ersten Rcaktionsschema.

Tabelle 1. NMR-Daten der Anionen von (3), gelöst in $\mathrm{CD}_{3} \mathrm{CN}$

${ }^{31} \mathrm{P}-\mathrm{NMR}\left(40.5 \mathrm{MHz}, \mathrm{H}_{3} \mathrm{PO}_{4}\right.$ extern, $\delta$ in $\left.\mathrm{ppm}\right)$

$\mathrm{R}=\mathrm{CH}_{3}: \quad \delta=+113.5$

$\mathrm{R}=\mathrm{C}_{6} \mathrm{H}_{5}: \quad \delta=+108.8$

\section{Arbeitsvorschrift:}

$\mathrm{Zu}$ äquivalenten Mengen von Phosphol und Triäthylamin in Diäthyläther tropft man langsam unter $\mathrm{N}_{2}$, Eiskühlung und Rühren eine ätherische Lösung von Brenzcatechin. Der ausfallende weiße Niederschlag wird in einer Umkehrfritte filtriert, mit Äther gewaschen und am Vakuum getrocknet. Ausbeute an sehr hygroskopischem (3) $>95 \%$

Eingegangen am 26. Februar 1973 [Z 811]

[1] D. Marquarding. F. Ramirez, 1. Ugi u. P. Gillespis. Angew. Chem. 85, 99 (1973): Angew. Chem. internat. Edit. 12, 91 (1973). 